\title{
The three-loop polarized singlet anomalous dimensions from off-shell operator matrix elements
}

\author{
J. Blümlein, ${ }^{a}$ P. Marquard, ${ }^{a}$ C. Schneider ${ }^{b}$ and K. Schönwald ${ }^{c}$ \\ ${ }^{a}$ Deutsches Elektronen-Synchrotron DESY, \\ Platanenallee 6, 15738 Zeuthen, Germany \\ ${ }^{b}$ Johannes Kepler University Linz, Research Institute for Symbolic Computation (RISC), \\ Altenberger Straße 69, A-4040 Linz, Austria \\ ${ }^{c}$ Institut für Theoretische Teilchenphysik, Karlsruher Institut für Technologie (KIT), \\ D-76128 Karlsruhe, Germany \\ E-mail: johannes.bluemlein@desy.de, peter.marquard@desy.de, \\ cschneid@risc.jku.at, kay.schoenwald@kit.edu
}

ABSTRACT: Future high luminosity polarized deep-inelastic scattering experiments will improve both the knowledge of the spin sub-structure of the nucleons and contribute further to the precision determination of the strong coupling constant, as well as, reveal currently yet unknown higher twist contributions in the polarized sector. For all these tasks to be performed, it is necessary to know the QCD leading twist scaling violations of the measured structure functions. Here an important ingredient consists in the polarized singlet anomalous dimensions and splitting functions in QCD. We recalculate these quantities to three-loop order in the M-scheme by using the traditional method of space-like offshell massless operator matrix elements, being a gauge-dependent framework. Here one obtains the anomalous dimensions without referring to gravitational currents, needed when calculating them using the forward Compton amplitude. We also calculate the non-singlet splitting function $\Delta P_{\mathrm{qq}}^{(2), \mathrm{s}, \mathrm{NS}}$ and compare the final results to the literature, also including predictions for the region of small values of Bjorken $x$.

KEYwords: Perturbative QCD, Renormalization Group

ArXiv ePrint: 2111.12401 


\section{Contents}

1 Introduction

2 The unrenormalized polarized operator matrix elements and details of the calculation

3 The polarized non-singlet anomalous dimension $\Delta \gamma_{\mathrm{qq}}^{(2), s, \mathrm{NS}} \quad 7$

4 The polarized singlet anomalous dimensions $\quad 8$

5 Comparison to the literature $\quad 19$

6 Conclusions 21

$\begin{array}{ll}\text { A A new polarized gluonic Feynman rule } & 21\end{array}$

\section{Introduction}

Polarized deep-inelastic scattering allows to reveal the spin and angular momentum structure of nucleons [1]. At sufficiently high scales of the virtuality of the exchanged electroweak current $Q^{2} \gg 1 \mathrm{GeV}^{2}$ and for not too small or too large values of Bjorken $x=$ $Q^{2} /(2 p . q)[2]$ the twist-2 contributions dominate over the higher twist contributions [3, 4] and target mass corrections $[5,6]$. Because of their strong variation, also the detailed control of the QED radiative corrections is required [7-10].

The scaling violations of the polarized deep-inelastic structure functions at the level of leading twist $\tau=2$ are determined by the anomalous dimensions of the composite quark and gluon operators [11-14] through the evolution of the polarized parton densities and the massless and massive Wilson coefficients. A major goal in measuring the polarized deepinelastic scattering process to high precision, e.g. at the EIC in the future [15] consist in the detailed measurement of the polarized parton distribution functions at a reference scale $Q_{0}^{2}$ together with the correlated measurement of the strong coupling constant $a_{s}\left(M_{Z}^{2}\right)=$ $\alpha_{s}\left(M_{Z}^{2}\right) /(4 \pi)$ [16-18]. One of the important ingredients to this is the knowledge of the polarized singlet anomalous dimensions to highest possible order. The polarized nonsinglet anomalous dimensions are known to 3-loop order $[19,20]$ and the polarized singlet anomalous dimensions were calculated to first [21-25], second [26-29] and third order in [30, 31], using different computation techniques. In ref. [30] the calculation has been performed using the forward Compton amplitude, which required reference to gravitational subsidiary currents for the gluonic sector. A direct calculation of the contributions $\propto T_{F},{ }^{1}$ of the threeloop anomalous dimensions is possible using massive on-shell operator matrix elements [31], without reference to gravitational currents. However, this method only allows to calculate the anomalous dimensions $\Delta \gamma_{q q}^{(2), \mathrm{PS}}$ and $\Delta \gamma_{q g}^{(2)}$ in complete form.

In the present paper we use the traditional approach of massless off-shell operator matrix elements (OMEs), allowing the direct calculation, as before for the non-singlet case

\footnotetext{
${ }^{1}$ The color factors are defined in [20], below eq. (22).
} 
and the three-loop anomalous dimensions of transversity in ref. [20]. However, for this method the computational framework is gauge dependent. Still the anomalous dimensions in minimal subtraction schemes remain gauge invariant. Specific physical projectors have to be used, cf. also [29]. In the polarized case, unlike the unpolarized case [32], there is no mixing with the so-called alien operators [29, 32-44]. The main goal of the present paper is the re-calculation of all three-loop polarized singlet anomalous dimensions by a different method, being the first re-calculation of the polarized anomalous dimensions $\Delta \gamma_{g q}^{(2)}$ and $\Delta \gamma_{g g}^{(2)}$. To some extent different steps in the computation technology are the same as in the non-singlet case, cf. [20] which will be referred to this paper and we only will describe new additional elements in the present paper. ${ }^{2}$ At the phenomenological side we mention that, unlike the singlet case, the flavor non-singlet description of the structure functions $g_{1,2}^{\mathrm{NS}}\left(x, Q^{2}\right)$ is well explored to three-loop order, also including the heavy flavor contributions [45-47].

The paper is organized as follows. In section 2 we derive the structure of the physical part of the flavor singlet polarized unrenormalized off-shell OMEs to three-loop order. From their pole terms of $O(1 / \varepsilon)$ one can extract the singlet anomalous dimensions. We work in the Larin scheme $[29,48]$ and perform finally the transformation to the M-scheme $[29,30]$. We also present the calculation details. Here a central method being applied is the method of arbitrary high moments [49]. In section 3 we calculate also the polarized non-singlet anomalous dimension $\Delta \gamma_{q q}^{(2), \mathrm{s}, \mathrm{NS}}$, cf. also [50], and present the polarized singlet anomalous dimensions in section 4. Comparisons to the literature are given in section 5, including a discussion of the small $x$ limit. Section 6 contains the conclusions and a new Feynman rule is presented in appendix A.

\section{The unrenormalized polarized operator matrix elements and details of the calculation}

The massless off-shell singlet OMEs are defined as expectation values of the local operators

$$
\begin{aligned}
& O_{q ; \mu_{1} \ldots \mu_{N}}^{\mathrm{S}, 5}=i^{N-1} \mathbf{S}\left[\bar{\psi} \gamma_{5} \gamma_{\mu_{1}} D_{\mu_{2}} \ldots D_{\mu_{N}} \psi\right]-\text { trace terms, } \\
& O_{g ; \mu_{1} \ldots \mu_{N}}^{\mathrm{S}, 5}=2 i^{N-2} \mathbf{S} \operatorname{tr}\left[\frac{1}{2} \varepsilon^{\mu_{1} \alpha \beta \gamma} F_{\beta \gamma}^{a} D_{\mu_{2}} \ldots D_{\mu_{N-1}} F_{\alpha, a}^{\mu_{N}}\right]-\text { trace terms }
\end{aligned}
$$

between quark (antiquark) $\psi(\bar{\psi})$ and gluonic states $F_{\mu_{1} \alpha}^{a}$ of space-like momentum $p, p^{2}<0$. The OMEs are given by

$$
\Delta \hat{A}_{i j}=\left\langle q(p), j\left|O_{i}^{\mathrm{S},(5)}\right| q(p), j\right\rangle
$$

For the other definitions, here and in the following, we refer the reader to ref. [20].

\footnotetext{
${ }^{2}$ This seems to be necessary to avoid critique by word processing codes, like e.g. iThenticate, even on the expense to not provide more comprehensive information to the reader in the same publication, which we regret.
} 
The Feynman rules of both QCD and of the local composite operators are given in [31, $51,52]$ and have to be extended by that of the polarized local five-gluon vertex, given in appendix A.

We use the Larin scheme $[48]^{3}$ to describe $\gamma^{5}$ in $D=4+\varepsilon$-dimensions and express $\gamma^{5}$ by

$$
\begin{aligned}
\gamma^{5} & =\frac{i}{24} \varepsilon_{\mu \nu \rho \sigma} \gamma^{\mu} \gamma^{\nu} \gamma^{\rho} \gamma^{\sigma}, \\
\not \gamma^{5} & =\frac{i}{6} \varepsilon_{\mu \nu \rho \sigma} \Delta^{\mu} \gamma^{\nu} \gamma^{\rho} \gamma^{\sigma} .
\end{aligned}
$$

The Levi-Civita symbols are now contracted in $D$ dimensions,

$$
\varepsilon_{\mu \nu \rho \sigma} \varepsilon^{\alpha \lambda \tau \gamma}=-\operatorname{Det}\left[g_{\omega}^{\beta}\right], \quad \beta=\alpha, \lambda, \tau, \gamma ; \quad \omega=\mu, \nu, \rho, \sigma .
$$

The Larin scheme is one of the consistent calculation schemes in the polarized case. We will later transform to another scheme, the M-scheme. It is needless to say that the calculation of any observable requires to calculate also the Wilson coefficients in the same scheme, which implies that the extracted twist-2 parton density functions are obtained in this scheme, despite being universal w.r.t. the scattering processes in which they are used. Any analytic continuation of $\gamma_{5}$ or the Levi-Civita symbol to $D$ dimensions violates Ward identities. One way to restore these consists in the calculation of scheme-invariant quantities.

Let us add a few remarks on the M-scheme, cf. [29]. It is one of the consistent schemes for dealing with $\gamma_{5}$ in $D$ dimensions. Historically, it has been motivated because here the supersymmetric relations hold to two-loop order [26-29], which are, however, not valid anymore at three-loop order, cf. (4.95) below and ref. [30]. The presentation of our results in the M-scheme has the reason to compare with the results of ref. [30]. Finally, one will perform the evolution of polarized structure functions in scheme-invariant form, as e.g. by studying the pair of observables $g_{1}\left(x, Q^{2}\right), \partial g_{1}\left(x, Q^{2}\right) / \partial \ln \left(Q^{2}\right)$, cf. [69, 70], or using one of the variety of the so-called DIS schemes, [71], cf. e.g. [72], to define the polarized parton densities, representing $g_{1}\left(x, Q^{2}\right)$ by purely quarkonic contributions, as at tree level.

The polarized operator matrix elements have the representation

$$
\begin{aligned}
\Delta \hat{A}_{q q}^{\mathrm{PS}} & =\left[\gamma_{5} \not \Delta \hat{A}_{q q}^{\mathrm{PS}, \mathrm{phys}}+\gamma_{5} \not p \frac{\Delta \cdot p}{p^{2}} \Delta \hat{A}_{q q}^{\mathrm{PS}, \mathrm{EOM}}\right](\Delta \cdot p)^{N-1} \\
\Delta \hat{A}_{q g} & =\varepsilon_{\mu \nu \alpha \beta} \Delta^{\alpha} p^{\beta} \frac{1}{\Delta \cdot p} \Delta \hat{A}_{q g}^{\mathrm{phys}}(\Delta \cdot p)^{N-1} \\
\Delta \hat{A}_{g q} & =\left[\gamma_{5} \not \Delta \hat{A}_{g q}^{\mathrm{phys}}+\gamma_{5} \not \frac{\Delta \cdot p}{p^{2}} \hat{A}_{g q}^{\mathrm{EOM}}\right](\Delta \cdot p)^{N-1} \\
\Delta \hat{A}_{g g, \mu \nu} & =\varepsilon_{\mu \nu \alpha \beta} \Delta^{\alpha} p^{\beta} \frac{1}{\Delta \cdot p} \Delta \hat{A}_{g q}^{\mathrm{phys}}(\Delta \cdot p)^{N-1},
\end{aligned}
$$

\footnotetext{
${ }^{3}$ See refs. [53-58] for other schemes and refs. [59-68] concerning the finite renormalization in different approaches.
} 
with $\Delta$ obeying $\Delta . \Delta=0$. It is convenient to introduce the following projectors on the physical part (phys)

$$
\begin{aligned}
\Delta \hat{A}_{i q}^{\text {phys }}= & -\frac{1}{4(D-2)(D-3)} \varepsilon_{\mu \nu \rho \sigma} p^{\rho} \Delta^{\sigma} \operatorname{tr}\left[\not p \gamma^{\mu} \gamma^{\nu} \Delta \hat{A}_{i q}\right](\Delta \cdot p)^{-N-1} \\
& -\frac{p^{2}}{4(D-2)(D-3)}(\Delta \cdot p)^{-N-2} \varepsilon_{\mu \nu \rho \sigma} p^{\rho} \Delta^{\sigma} \operatorname{tr}\left[\Delta \gamma^{\mu} \gamma^{\nu} \Delta \hat{A}_{i q}\right] \\
\Delta \hat{A}_{i g}^{\text {phys }}= & \frac{1}{(D-2)(D-3)} \varepsilon_{\mu \nu \rho \sigma} \Delta^{\rho} p^{\sigma}(\Delta \cdot p)^{-N-1} \Delta \hat{A}_{i g}^{\mu \nu},
\end{aligned}
$$

with $i=q, g$. Only these contributions are necessary for the calculation of the singlet anomalous dimensions. In Mellin $N$ space the following representation holds

$$
\Delta \hat{A}_{i j}=\delta_{i j}+\sum_{k=1}^{\infty} \hat{a}^{k} S_{\varepsilon}^{k}\left(\frac{-p^{2}}{\mu^{2}}\right)^{\varepsilon k / 2} \Delta \hat{A}_{i j}^{(k)},
$$

with the spherical factor $S_{\varepsilon}$ given in eq. (13) of [20]. The bare coupling constant $\hat{a}$ and the gauge parameter $\xi$ are defined in eqs. (15)-(22) of [20], working in the $R_{\hat{\xi}}$ gauge, by using relations from [73-87].

In Mellin $N$ space the $Z$-factor of a local singlet operator reads [52]

$$
\begin{aligned}
Z_{i j}^{\mathrm{S}}= & \delta_{i j}+a \frac{\Delta \gamma_{i j}^{(0)}}{\varepsilon}+a^{2}\left[\frac{1}{\varepsilon^{2}}\left(\frac{1}{2} \Delta \gamma_{i l}^{(0)} \Delta \gamma_{l j}^{(0)}+\beta_{0} \Delta \gamma_{i j}^{(0)}\right)+\frac{1}{2 \varepsilon} \Delta \gamma_{i j}^{(1)}\right] \\
& +a^{3}\left[\frac{1}{\varepsilon^{3}}\left(\frac{1}{6} \Delta \gamma_{i l}^{(0)} \Delta \gamma_{l k}^{(0)} \Delta \gamma_{k j}^{(0)}+\beta_{0} \Delta \gamma_{i l}^{(0)} \Delta \gamma_{l j}^{(0)}+\frac{4}{3} \beta_{0}^{2} \Delta \gamma_{i j}^{(0)}\right)\right. \\
& \left.+\frac{1}{\varepsilon^{2}}\left(\frac{1}{6} \Delta \gamma_{i l}^{(1)} \Delta \gamma_{l j}^{(0)}+\frac{1}{3} \Delta \gamma_{i l}^{(0)} \Delta \gamma_{l j}^{(1)}+\frac{2}{3} \beta_{0} \Delta \gamma_{i j}^{(1)}+\frac{2}{3} \beta_{1} \Delta \gamma_{i j}^{(0)}\right)+\frac{1}{3 \varepsilon} \Delta \gamma_{i j}^{(2)}\right]
\end{aligned}
$$

In (2.14) the terms $\Delta \gamma_{i j}^{(k)}, \quad k=0,1,2, \ldots$ denote the expansion coefficients of the anomalous dimension

$$
\Delta \gamma_{i j}=\sum_{k=1}^{\infty} a^{k} \Delta \gamma_{i j}^{(k-1)}
$$

The partly renormalized polarized singlet OMEs, $\Delta \tilde{A}_{i j}^{\text {phys }}$, read

$$
\begin{aligned}
\Delta \tilde{A}_{i j}^{\text {phys }}= & 1+a\left[\frac{a_{i j}^{(1,-1)}}{\varepsilon}+a_{i j}^{(1,0)}+a_{i j}^{(1,1)} \varepsilon\right]+a^{2}\left[\frac{a_{i j}^{(2,-2)}}{\varepsilon^{2}}+\frac{a_{i j}^{(2,-1)}}{\varepsilon}+a_{i j}^{(2,0)}\right] \\
& +a^{3}\left[\frac{a_{i j}^{(3,-3)}}{\varepsilon^{3}}+\frac{a_{i j}^{(3,-2)}}{\varepsilon^{2}}+\frac{a_{i j}^{(3,-1)}}{\varepsilon}\right] .
\end{aligned}
$$

The expansion coefficients $a_{i j}^{(\mathrm{k}, \mathrm{l})}$ are gauge dependent in general. The renormalized OMEs in the Larin scheme are given by

$$
\Delta A_{i j}^{\text {phys }}=\left(\Delta Z_{i k}^{S}\right)^{-1} \Delta \tilde{A}_{k j}^{\text {phys }},
$$


expanded to $O\left(a^{3}\right)$ and setting $S_{\varepsilon}=1$. The anomalous dimensions are iteratively extracted from the $1 / \varepsilon$ pole terms and the other expansion coefficients $a_{i j}^{(k, l)}$ are given in ref. [88].

The anomalous dimensions in the M-scheme $[29,30]^{4}$ are obtained by the following transformations

$$
\begin{aligned}
\Delta \gamma_{i j}^{(0), \mathrm{M}} & =\Delta \gamma_{i j}^{(0), \mathrm{L}}, \\
\Delta \gamma_{q q}^{(1), \mathrm{NS}, \mathrm{M}} & =\Delta \gamma_{q q}^{(1), \mathrm{NS}, \mathrm{L}}+2 \beta_{0} z_{q q}^{(1)}, \\
\Delta \gamma_{q q}^{(1), \mathrm{PS}, \mathrm{M}} & =\Delta \gamma_{q q}^{(1), \mathrm{PS}, \mathrm{L}}, \\
\Delta \gamma_{q g}^{(1), \mathrm{M}} & =\Delta \gamma_{q g}^{(1), \mathrm{L}}+\Delta \gamma_{q g}^{(0)} z_{q q}^{(1)}, \\
\Delta \gamma_{g q}^{(1), \mathrm{M}} & =\Delta \gamma_{g q}^{(1), \mathrm{L}}-\Delta \gamma_{g q}^{(0)} z_{q q}^{(1)}, \\
\Delta \gamma_{g g}^{(1), \mathrm{M}} & =\Delta \gamma_{g g}^{(1), \mathrm{L}}, \\
\Delta \gamma_{q q}^{(2), \mathrm{NS}, \mathrm{M}} & =\Delta \gamma_{q q}^{(2), \mathrm{NS}, \mathrm{L}}-2 \beta_{0}\left(\left(z_{q q}^{(1)}\right)^{2}-2 z_{q q}^{(2), \mathrm{NS}}\right)+2 \beta_{1} z_{q q}^{(1)}, \\
\Delta \gamma_{q q}^{(2), \mathrm{PS}, \mathrm{M}} & =\Delta \gamma_{q q}^{(2), \mathrm{PS}, \mathrm{L}}+4 \beta_{0} z_{q q}^{(2), \mathrm{PS}}, \\
\Delta \gamma_{q g}^{(2), \mathrm{M}} & =\Delta \gamma_{q g}^{(2), \mathrm{L}}+\Delta \gamma_{q g}^{(1), \mathrm{M}} z_{q q}^{(1)}+\Delta \gamma_{q g}^{(0)}\left(z_{q q}^{(2)}-\left(z_{q q}^{(1)}\right)^{2}\right), \\
\Delta \gamma_{g q}^{(2), \mathrm{M}} & =\Delta \gamma_{g q}^{(2), \mathrm{L}}-\Delta \gamma_{g q}^{(1), \mathrm{M}} z_{q q}^{(1)}-\Delta \gamma_{g q}^{(0)} z_{q q}^{(2)}, \\
\Delta \gamma_{g g}^{(2), \mathrm{M}} & =\Delta \gamma_{g g}^{(2), \mathrm{L}},
\end{aligned}
$$

with [29]

$$
\begin{aligned}
z_{q q}^{(1)}= & -\frac{8 C_{F}}{N(N+1)}, \\
z_{q q}^{(2), \mathrm{NS}}= & C_{F} T_{F} N_{F} \frac{16\left(-3-N+5 N^{2}\right)}{9 N^{2}(1+N)^{2}}+C_{A} C_{F}\left\{-\frac{4 R_{1}}{9 N^{3}(1+N)^{3}}-\frac{16}{N(1+N)} S_{-2}\right\} \\
& +C_{F}^{2}\left\{\frac{8\left(2+5 N+8 N^{2}+N^{3}+2 N^{4}\right)}{N^{3}(1+N)^{3}}+\frac{16(1+2 N)}{N^{2}(1+N)^{2}} S_{1}\right. \\
& \left.+\frac{16}{N(1+N)} S_{2}+\frac{32}{N(1+N)} S_{-2}\right\} \\
z_{q q}^{(2), \mathrm{PS}}= & 8 C_{F} T_{F} N_{F} \frac{(N+2)\left(1+N-N^{2}\right)}{N^{3}(N+1)^{3}} \\
z_{q q}^{(2)}= & z_{q q}^{(2), \mathrm{NS}}+z_{q q}^{(2), \mathrm{PS}}
\end{aligned}
$$

and

$$
R_{1}=103 N^{4}+140 N^{3}+58 N^{2}+21 N+36 .
$$

Let us now turn to the technical aspects of the present calculation, which has been widely automated and the corresponding chain of programs has been described to some extent in our previous paper [20]. A main point concerns the observation of the current

\footnotetext{
${ }^{4}$ We reproduce these transformations here, because they are of instrumental importance for the understanding of the present calculation, which is performed in the Larin scheme.
} 
crossing relations in the present case, cf. [11, 89] and also ref. [88], projecting onto the contributions of the odd integer moments

$$
\sum_{N=0}^{\infty}(\Delta . k)^{N}\left(t^{N}-(-t)^{N}\right) \rightarrow\left[\frac{1}{1-\Delta \cdot k t}-\frac{1}{1+\Delta \cdot k t}\right] .
$$

Through this one obtains a quadratic dependence on the resummation variable $t$. An expansion in $t$ leads to the moments again.

Our chain of programs, from the generation of Feynman diagrams to the final result, the polarized anomalous dimensions at three-loop order includes the packages QGRAF, FORM, Color, EvaluateMultiSums, Crusher, SolveCoupledSystems, Guess, Sage, Sigma, HarmoncisSums [52, 90-123]. More details were given in ref. [20]. We determine the contributions to the anomalous dimensions due to the different color and multiple zeta values [122] individually. In the unfolding process also higher order terms $a_{i j}^{(k)}$ in the dimensional parameter $\varepsilon$ are needed to be calculated, cf. ref. [88]. We use the integrationby-parts relations $[98,124-128]$ to reduce the problem to master integrals. The method of arbitrary high moments [49] allows to find the needed difference equations, which are then solved using algorithms in difference ring theory [129-141]. To calculate initial values for the solution we use relations given in [142-147].

We turn now to some statistical characteristics of the present calculation. In the polarized flavor singlet case 125 irreducible diagrams contribute for $\Delta A_{q q}^{(3), \mathrm{PS}}, 1101$ for $\Delta A_{q g}^{(3)}$, 400 for $\Delta A_{g q}^{(3)}$, and 1598 for $\Delta A_{g g}^{(3)}$. For comparison the number of diagrams for $\Delta A_{q q}^{(3), \text { NS }}$ amounts to 559. Finally, 24 diagrams contribute to the part of the forward Compton amplitude, from which $\Delta \gamma_{q q}^{(2), \mathrm{s}, \mathrm{NS}}$ is extracted. The total number of irreducible diagrams in the polarized singlet case at three-loop order is larger by a factor of $\sim 22$ than in the two-loop order. The reducible diagrams are accounted for by wave-function renormalization $[84,86,87]$, decorating the OMEs at lower order in the coupling constant $[29,88]$.

To calculate the anomalous dimensions $\Delta \gamma_{\mathrm{ij}}^{(2)}$ we generated 3000 odd moments. It turns out that the determination of the largest recurrence requires 462 moments for $\Delta \gamma_{\mathrm{qq}}^{(2), \mathrm{PS}}$, 989 moments for $\Delta \gamma_{\mathrm{qg}}^{(2)}, 1035$ moments for $\Delta \gamma_{\mathrm{gq}}^{(2)}, 1568$ moments for $\Delta \gamma_{\mathrm{gg}}^{(2)}$.

In this way the anomalous dimensions are obtained. The largest difference equation contributing has order $\mathrm{o}=16$ and degree $\mathrm{d}=304$. These numbers are of the order obtained in the non-singlet case in ref. [101], where the largest difference equation contributing had order $\mathrm{o}=16$ and degree $\mathrm{d}=192$, and required 1079 moments. A moment based test-run for the polarized anomalous dimensions, like in the unpolarized case, has not been performed.

The overall computation time using the automated chain of codes described amounted to about 18 days of CPU time on Intel(R) Xeon(R) CPU E5-2643 v4 processors. Again we have only retained the first power of the gauge parameter to have a first check on the renormalization.

The anomalous dimensions, $\Delta \gamma_{\mathrm{ij}}$, can all be expressed by harmonic sums $[106,107]$, defined in eq. (34) of ref. [20]. Their Mellin inversion to the splitting functions $\Delta P_{i j}(z)$

$$
\Delta \gamma_{i j}(N)=-\int_{0}^{1} d z z^{N-1} \Delta P_{i j}(z)
$$


is obtained using routines of the packages HarmonicSums and are expressed by harmonic polylogarithms [108], which are defined in eqs. (36), (37) of ref. [20].

Because of the different requirements on the respective integrals, one distinguishes three contributions to the individual splitting functions in $z$-space

$$
\Delta P(z)=\Delta P^{\delta}(z)+\Delta P^{\mathrm{plu}}(z)+\Delta P^{\mathrm{reg}}(z)
$$

which are defined in eqs. (38), (39) of ref. [20].

Also here we reduce the expressions to the algebraic basis, cf. [121], which has the advantage that only the minimal set has to be calculated in numerical applications [148150]. We will not present the splitting function in explicit form, since the expressions are rather lengthy. They are given in computer-readable form in the supplementary material. The polarized singlet anomalous dimensions are given in section 4 .

\section{The polarized non-singlet anomalous dimension $\Delta \gamma_{\mathrm{qq}}^{(2), s, \mathrm{NS}}$}

In our previous paper [20] we had not yet calculated the non-singlet anomalous dimension $\Delta \gamma_{\mathrm{qq}}^{(2), s, \mathrm{NS}}$, which emerges from three-loop order onward. It is best calculated using the vector-axialvector interference term in the forward Compton amplitude, corresponding to the associated structure function $g_{5}^{-}\left(x, Q^{2}\right)$, ref. [89], corresponding to the difference in case of $W^{-}$and $W^{+}$charged current scattering. Due to its crossing relations it has even moments. This anomalous dimension has been calculated previously in ref. [50].

The corresponding gauge boson vertex is parameterized by

$$
i\left[v \gamma_{\mu}+a \frac{i}{6} \varepsilon_{\mu \nu_{1} \nu_{2} \nu_{3}} \gamma^{\nu_{1}} \gamma^{\nu_{2}} \gamma^{\nu_{3}}\right]
$$

and we consider the current interference term $\propto a \cdot v$. The projectors for the massless external quark lines of momentum $p$ and boson lines corresponding to a tensor of rank two read

$$
P^{q}=\frac{1}{4} \operatorname{tr}\left[\frac{i}{6} \varepsilon_{p \rho_{1} \rho_{2} \rho_{3}} \gamma^{\rho_{1}} \gamma^{\rho_{2}} \gamma^{\rho_{3}} \ldots\right] ; \quad P_{\mu \nu}^{b}=-\frac{g_{\mu \nu}}{D-1} .
$$

The forward Compton amplitude depends on the invariants $Q^{2}=-q^{2}$ and $p . q=Q^{2} /(2 z) \equiv$ $\left(Q^{2} / 2\right) y$. The diagrams can be represented as formal power series in $y$

$$
F(y)=\sum_{N=0}^{\infty} f(N) y^{N}
$$

cf. e.g. [20], section 2. The IBP-reduction in this case leads to three families and 101 master integrals in total. We consider the differential equations for the individual master integrals $M_{k}(y, \varepsilon)$, which are computed using the method described in [151]. After insertion of the master integrals into the amplitude and subsequent expansion in the dimensional parameter $\varepsilon$, the anomalous dimension can be determined by using the command GetMoment $[\mathrm{F}[\mathrm{y}], \mathrm{y}, \mathrm{N}]$ of the package HarmonicSums from the pole term $O(1 / \varepsilon)$ of the 
forward Compton amplitude. We obtain

$$
\begin{aligned}
\Delta \gamma_{\mathrm{NS}}^{(2), \mathrm{s}}= & -16 \frac{1+(-1)^{N}}{2} N_{F} \frac{d^{a b c} d_{a b c}}{N_{c}}\left[\frac{1}{N^{2}}+\frac{2 Q_{1}}{N^{4}(1+N)^{4}} S_{1}+\frac{2\left(2+3 N+3 N^{2}\right)}{N^{2}(1+N)^{2}}\right. \\
& \times\left[S_{3}-2 S_{-3}+4 S_{-2,1}\right]+\left(\frac{4\left(2+4 N+4 N^{2}+N^{3}+N^{4}\right)}{N^{3}(1+N)^{3}}+\frac{8(-1+N)(2+N)}{N^{2}(1+N)^{2}} S_{1}\right) \\
& \left.\times S_{-2}\right]
\end{aligned}
$$

with

$$
Q_{1}=3 N^{6}+8 N^{5}-N^{4}-14 N^{3}-29 N^{2}-21 N-6 .
$$

The corresponding splitting function reads

$$
\begin{aligned}
\Delta P_{\mathrm{NS}}^{(2), \mathrm{s}}= & 16 N_{F} \frac{d^{a b c} d_{a b c}}{N_{c}}\left\{( 1 + x ) \left[-4 \mathrm{H}_{0} \mathrm{H}_{-1}\left(-4+5 \mathrm{H}_{-1}\right)+8 \mathrm{H}_{0,-1}\left(-2+5 \mathrm{H}_{-1}\right)\right.\right. \\
& +18 \mathrm{H}_{-1} \mathrm{H}_{0}^{2}+\left(-2 \mathrm{H}_{0}^{2}+32 \mathrm{H}_{-1}\right) \mathrm{H}_{0,1}+8 \mathrm{H}_{0} \mathrm{H}_{0,0,1}-32 \mathrm{H}_{0,1,-1}-32 \mathrm{H}_{0,-1,1} \\
& \left.-40 \mathrm{H}_{0,-1,-1}-\left(52 \mathrm{H}_{-1}+8 \mathrm{H}_{0,1}\right) \zeta_{2}\right]+(1-x)\left[24 \mathrm{H}_{1}-\mathrm{H}_{0}^{2} \mathrm{H}_{1}+2 \mathrm{H}_{0} \mathrm{H}_{0,1}\right. \\
& \left.+4 \mathrm{H}_{0}^{2} \mathrm{H}_{0,-1}+8 \mathrm{H}_{0,-1}^{2}-8 \mathrm{H}_{0} \mathrm{H}_{0,0,-1}-16 \mathrm{H}_{0} \mathrm{H}_{0,-1,-1}-\left(20 \mathrm{H}_{1}+8 \mathrm{H}_{0,-1}\right) \zeta_{2}\right] \\
& -(1+24 x) \mathrm{H}_{0}-4 x \mathrm{H}_{0}^{2}-6 x \mathrm{H}_{0}^{3}+\frac{1}{3} x \mathrm{H}_{0}^{4}+2(5+4 x) \mathrm{H}_{0,1}-28 \mathrm{H}_{0} \mathrm{H}_{0,-1} \\
& +4(1-9 x) \mathrm{H}_{0,0,1}+4(5-9 x) \mathrm{H}_{0,0,-1}+\left(2(-5+4 x)+2(-3+37 x) \mathrm{H}_{0}\right. \\
& \left.\left.-2(3+5 x) \mathrm{H}_{0}^{2}\right) \zeta_{2}+\left(2(3+44 x)-16 x \mathrm{H}_{0}\right) \zeta_{3}+2(5+3 x) \zeta_{2}^{2}\right\} .
\end{aligned}
$$

Here $\zeta_{k}$ denotes the Riemann $\zeta$-function at integer argument $k \geq 2$ and the color factor is normalized in the present case to $d^{a b c} d_{a b c} / N_{c}=5 / 18$ in QCD. The leading small $z$ contribution of $\Delta P_{\mathrm{NS}}^{(2), \mathrm{s}}$ is $\propto \ln ^{2}(z)$. This behaviour is, however, not dominant in kinematic regions being accessible at present. The asymptotic behaviour reaches the complete function up to $10 \%$ below $z \sim 10^{-11}$ only. One more logarithmic order allows a description below $z \sim 10^{-3}$.

\section{The polarized singlet anomalous dimensions}

In the following we use the minimal representations in terms of the contributing harmonic sums and harmonic polylogarithms by applying the algebraic relations [121] between the harmonic sums and the harmonic polylogarithms. The polarized singlet anomalous dimensions can be represented by the following 23 harmonic sums up to weight $w=5$ to three-loop order

$\left\{S_{-5}, S_{-4}, S_{-3}, S_{-2}, S_{1}, S_{2}, S_{3}, S_{4}, S_{5}, S_{-4,1}, S_{-3,1}, S_{-2,1}, S_{-2,2}, S_{-2,3}, S_{2,-3}, S_{2,1}, S_{3,1}, S_{-3,1,1}\right.$, $\left.S_{-2,1,1}, S_{-2,2,1}, S_{2,1,-2}, S_{2,1,1}, S_{-2,1,1,1}\right\}$. 
This number is further reduced using also the structural relations $[123,152]$ to at least 15 sums. In the present case the 10 sums

$$
\left\{S_{1}, S_{2,1}, S_{-2,1}, S_{-3,1}, S_{-4,1}, S_{2,1,1}, S_{-2,1,1} \cdot S_{2,1,-2}, S_{-3,1,1}, S_{-2,1,1,1}\right\}
$$

suffice.

The splitting functions in $z$-space depend on the 26 harmonic polylogarithms

$$
\begin{aligned}
& \left\{\mathrm{H}_{-1}, \mathrm{H}_{0}, \mathrm{H}_{1}, \mathrm{H}_{0,-1}, \mathrm{H}_{0,1}, \mathrm{H}_{0,-1,-1}, \mathrm{H}_{0,-1,1}, \mathrm{H}_{0,0,-1}, \mathrm{H}_{0,0,1}, \mathrm{H}_{0,1,-1}, \mathrm{H}_{0,1,1}, \mathrm{H}_{0,-1,-1,-1}, \mathrm{H}_{0,-1,-1,1},\right. \\
& \mathrm{H}_{0,-1,0,1}, \mathrm{H}_{0,-1,1,-1}, \mathrm{H}_{0,-1,1,1}, \mathrm{H}_{0,0,-1,-1}, \mathrm{H}_{0,0,-1,1}, \mathrm{H}_{0,0,0,-1}, \mathrm{H}_{0,0,0,1}, \mathrm{H}_{0,0,1,-1}, \mathrm{H}_{0,0,1,1}, \mathrm{H}_{0,1,-1,-1}, \\
& \left.\mathrm{H}_{0,1,-1,1}, \mathrm{H}_{0,1,1,-1}, \mathrm{H}_{0,1,1,1}\right\}
\end{aligned}
$$

The harmonic sums are defined at the odd integers in the first place and the analytic continuation to $N \in \mathbb{C}$ is performed from there, [123, 153-156].

We obtain the following expressions for the polarized singlet anomalous dimensions in Mellin- $N$ space, using the shorthand notation $S_{\vec{a}}(N) \equiv S_{\vec{a}}$ from one- to three-loop order. Here we dropped the prefactor $\frac{1}{2}\left(1-(-1)^{N}\right)$.

$$
\begin{aligned}
\Delta \gamma_{q q}^{(0)}= & C_{F}\left[-\frac{2\left(2+3 N+3 N^{2}\right)}{N(1+N)}+8 S_{1}\right], \\
\Delta \gamma_{q g}^{(0)}= & -T_{F} N_{F} \frac{8(N-1)}{N(1+N)}, \\
\Delta \gamma_{g q}^{(0)}= & -C_{F} \frac{4(2+N)}{N(1+N)}, \\
\Delta \gamma_{g g}^{(0)}= & T_{F} N_{F} \frac{8}{3}+C_{A}\left[-\frac{2\left(24+11 N+11 N^{2}\right)}{3 N(1+N)}+8 S_{1}\right], \\
\Delta \gamma_{q q}^{(1), \mathrm{PS}}= & C_{F} T_{F} N_{F} \frac{16(2+N)\left(1+2 N+N^{3}\right)}{N^{3}(1+N)^{3}}, \\
\Delta \gamma_{q g}^{(1)}= & C_{F} T_{F} N_{F}\left[-\frac{8(-1+N)\left(2-N+10 N^{3}+5 N^{4}\right)}{N^{3}(1+N)^{3}}+\frac{32(N-1)}{N^{2}(1+N)} S_{1}\right. \\
& \left.-\frac{16(N-1)}{N(1+N)}\left[S_{1}^{2}-S_{2}\right]\right]+C_{A} T_{F} N_{F}\left[-\frac{16 P_{20}}{N^{3}(1+N)^{3}}-\frac{64 S_{1}}{N(1+N)^{2}}\right. \\
& \left.+\frac{16(N-1)}{N(1+N)}\left[S_{1}^{2}+S_{2}+2 S_{-2}\right]\right], \\
\Delta \gamma_{g q}^{(1)}= & C_{F}\left[T_{F} N_{F}\left(\frac{32(2+N)(2+5 N)}{9 N(1+N)^{2}}-\frac{32(2+N)}{3 N(1+N)} S_{1}\right)+C_{A}\left(-\frac{8 P_{33}}{9 N^{3}(1+N)^{3}}\right.\right. \\
& \left.\left.+\frac{8\left(12+22 N+11 N^{2}\right) S_{1}}{3 N^{2}(1+N)}-\frac{8(2+N) S_{1}^{2}}{N(1+N)}+\frac{8(2+N) S_{2}}{N(1+N)}+\frac{16(2+N) S_{-2}}{N(1+N)}\right)\right]
\end{aligned}
$$




$$
\begin{aligned}
& +C_{F}^{2}\left[\frac{4(2+N)(1+3 N)\left(-2-N+3 N^{2}+3 N^{3}\right)}{N^{3}(1+N)^{3}}-\frac{8(2+N)(1+3 N)}{N(1+N)^{2}} S_{1}\right. \\
& \left.+\frac{8(2+N)}{N(1+N)}\left[S_{1}^{2}+S_{2}\right]\right] \\
& \Delta \gamma_{g g}^{(1)}=C_{F} T_{F} N_{F} \frac{8 P_{40}}{N^{3}(1+N)^{3}}+C_{A}^{2}\left[-\frac{4 P_{46}}{9 N^{3}(1+N)^{3}}+\left(\frac{8 P_{17}}{9 N^{2}(1+N)^{2}}-32 S_{2}\right) S_{1}\right. \\
& \left.+\frac{64}{N(1+N)} S_{2}-16 S_{3}+\left(\frac{64}{N(1+N)}-32 S_{1}\right) S_{-2}-16 S_{-3}+32 S_{-2,1}\right] \\
& +C_{A} T_{F} N_{F}\left[\frac{32 P_{5}}{9 N^{2}(1+N)^{2}}-\frac{160}{9} S_{1}\right] \text {, } \\
& \Delta \gamma_{q q}^{(2), \mathrm{PS}}=C_{F}\left[T _ { F } ^ { 2 } N _ { F } ^ { 2 } \left[-\frac{64(2+N) P_{30}}{27 N^{4}(1+N)^{4}}+\frac{64(2+N)\left(6+10 N-3 N^{2}+11 N^{3}\right)}{9 N^{3}(1+N)^{3}} S_{1}\right.\right. \\
& \left.-\frac{32(N-1)(2+N)}{3 N^{2}(1+N)^{2}}\left[S_{1}^{2}+S_{2}\right]\right]+C_{A} T_{F} N_{F}\left[\frac{8 P_{9}}{3 N^{3}(1+N)^{3}} S_{1}^{2}+\frac{8 P_{10}}{3 N^{3}(1+N)^{3}} S_{2}\right. \\
& +\frac{16 P_{61}}{27 N^{5}(1+N)^{5}}+\left(-\frac{16 P_{51}}{9 N^{4}(1+N)^{4}}+\frac{32(N-1)(2+N)}{N^{2}(1+N)^{2}} S_{2}\right) S_{1} \\
& -\frac{32(N-1)(2+N)}{3 N^{2}(1+N)^{2}} S_{1}^{3}+\frac{16\left(-58+23 N+23 N^{2}\right)}{3 N^{2}(1+N)^{2}} S_{3}+\left(-\frac{32 P_{1}}{N^{3}(1+N)^{3}}\right. \\
& \left.+\frac{64(N-1)(2+N)}{N^{2}(1+N)^{2}} S_{1}\right) S_{-2}+\frac{32\left(-10+7 N+7 N^{2}\right)}{N^{2}(1+N)^{2}} S_{-3}-\frac{64(N-1)(2+N)}{N^{2}(1+N)^{2}} S_{2,1} \\
& \left.\left.-\frac{64\left(-2+3 N+3 N^{2}\right)}{N^{2}(1+N)^{2}} S_{-2,1}-\frac{192(N-1)(2+N)}{N^{2}(1+N)^{2}} \zeta_{3}\right]\right] \\
& +C_{F}^{2} T_{F} N_{F}\left[-\frac{16(2+N) P_{54}}{N^{5}(1+N)^{5}}+\left(\frac{16(2+N) P_{27}}{N^{4}(1+N)^{4}}-\frac{32(N-1)(2+N)}{N^{2}(1+N)^{2}} S_{2}\right) S_{1}\right. \\
& -\frac{8(N-1)(2+N)\left(2+3 N+3 N^{2}\right)}{N^{3}(1+N)^{3}} S_{1}^{2}+\frac{32(N-1)(2+N)}{3 N^{2}(1+N)^{2}} S_{1}^{3} \\
& -\frac{8(2+N)\left(14+23 N+11 N^{3}\right)}{N^{3}(1+N)^{3}} S_{2}-\frac{224(N-1)(2+N)}{3 N^{2}(1+N)^{2}} S_{3} \\
& \left.+\frac{64(N-1)(2+N)}{N^{2}(1+N)^{2}} S_{2,1}+\frac{192(N-1)(2+N)}{N^{2}(1+N)^{2}} \zeta_{3}\right] \\
& \Delta \gamma_{q g}^{(2)}=C_{F} T_{F}^{2} N_{F}^{2}\left[\frac{4 P_{64}}{27 N^{5}(1+N)^{5}}+\left(-\frac{32\left(-24+4 N+47 N^{2}\right)}{27 N^{2}(1+N)}-\frac{32(N-1)}{3 N(1+N)} S_{2}\right) S_{1}\right. \\
& \left.+\frac{32(N-1)(3+10 N)}{9 N^{2}(1+N)} S_{1}^{2}-\frac{32(N-1)}{9 N(1+N)} S_{1}^{3}+\frac{32(5 N-1)}{3 N^{2}(1+N)} S_{2}+\frac{320(N-1)}{9 N(1+N)} S_{3}\right]
\end{aligned}
$$




$$
\begin{aligned}
& +C_{A} C_{F} T_{F} N_{F}\left[\frac{8 P_{29}}{3 N^{3}(1+N)^{3}} S_{2}+\frac{P_{65}}{27 N^{5}(1+N)^{5}(2+N)}+\left(-\frac{384(N-1)}{N(1+N)} S_{2,1}\right.\right. \\
& +\frac{16 P_{60}}{27 N^{4}(1+N)^{4}(2+N)}+\frac{16\left(75+14 N+18 N^{2}+N^{3}\right) S_{2}}{3 N^{2}(1+N)^{2}}+\frac{640(N-1)}{3 N(1+N)} S_{3} \\
& \left.-\frac{192(N-1)}{N(1+N)} \zeta_{3}\right) S_{1}+\left(-\frac{8 P_{25}}{9 N^{3}(1+N)^{3}}+\frac{160(N-1)}{N(1+N)} S_{2}\right) S_{1}^{2} \\
& +\frac{16\left(3-31 N-18 N^{2}+10 N^{3}\right)}{9 N^{2}(1+N)^{2}} S_{1}^{3}+\frac{32(N-1)}{3 N(1+N)} S_{1}^{4}-\frac{64(N-1)}{N(1+N)} S_{2}^{2} \\
& -\frac{16(N-1)\left(240-17 N+19 N^{2}\right)}{9 N^{2}(1+N)^{2}} S_{3}+\left(\frac{128(N-1)\left(-4-N+N^{2}\right)}{N^{2}(1+N)^{2}(2+N)} S_{1}\right. \\
& \left.-\frac{32 P_{24}}{N^{3}(1+N)^{3}(2+N)}+\frac{192(N-1)}{N(1+N)} S_{1}^{2}\right) S_{-2}+\frac{96(N-1)}{N(1+N)} S_{-2}^{2} \\
& +\frac{32(N-1)(2+N)(-1+3 N)}{N^{2}(1+N)^{2}} S_{-3}+\frac{96(N-1)\left(4+N+N^{2}\right)}{N^{2}(1+N)^{2}} S_{2,1} \\
& +\frac{160(N-1)}{N(1+N)} S_{-4}+\frac{64(N-1)}{N(1+N)} S_{3,1}-\frac{128(N-1)^{2}}{N^{2}(1+N)^{2}} S_{-2,1}+\frac{64(N-1)}{N(1+N)} S_{-2,2} \\
& \left.+\frac{192(N-1)}{N(1+N)} S_{2,1,1}-\frac{256(N-1)}{N(1+N)} S_{-2,1,1}-\frac{192(N-1)\left(-5+3 N+3 N^{2}\right)}{N^{2}(1+N)^{2}} \zeta_{3}\right] \\
& +C_{A} T_{F}^{2} N_{F}^{2}\left[\frac{16 P_{55}}{27 N^{4}(1+N)^{4}}+\left(\frac{64\left(23+50 N+10 N^{2}+19 N^{3}\right)}{27 N(1+N)^{3}}-\frac{32(N-1)}{3 N(1+N)}\right.\right. \\
& \left.\times S_{2}\right) S_{1}-\frac{64\left(-2+5 N^{2}\right)}{9 N(1+N)^{2}} S_{1}^{2}+\frac{32(N-1)}{9 N(1+N)} S_{1}^{3}-\frac{64\left(-2+6 N+5 N^{2}\right)}{9 N(1+N)^{2}} S_{2} \\
& \left.+\frac{64(N-1)}{9 N(1+N)} S_{3}-\frac{128(-2+5 N)}{9 N(1+N)} S_{-2}+\frac{128(N-1)}{3 N(1+N)}\left[S_{-3}+S_{2,1}\right]\right] \\
& +C_{A}^{2} N_{F} T_{F}\left[\frac{16 P_{34}}{9 N^{3}(1+N)^{3}} S_{2}-\frac{8 P_{70}}{27 N^{5}(1+N)^{5}(2+N)}+\left(-\frac{8 P_{56}}{27 N^{4}(1+N)^{4}}\right.\right. \\
& +\frac{8\left(-72+181 N-48 N^{2}+11 N^{3}\right)}{3 N^{2}(1+N)^{2}} S_{2}-\frac{704(N-1)}{3 N(1+N)} S_{3}+\frac{128(N-1)}{N(1+N)} S_{2,1} \\
& \left.+\frac{512(N-1)}{N(1+N)} S_{-2,1}+\frac{192(N-1)}{N(1+N)} \zeta_{3}\right) S_{1}+\left(\frac{16 P_{31}}{9 N^{3}(1+N)^{3}}-\frac{160(N-1)}{N(1+N)} S_{2}\right) S_{1}^{2} \\
& -\frac{8\left(-24-59 N+11 N^{3}\right)}{9 N^{2}(1+N)^{2}} S_{1}^{3}-\frac{16(N-1)}{3 N(1+N)} S_{1}^{4}-\frac{16(N-1)}{N(1+N)} S_{2}^{2} \\
& -\frac{16\left(345-428 N+11 N^{3}\right)}{9 N^{2}(1+N)^{2}} S_{3}-\frac{32(N-1)}{N(1+N)} S_{4}+\left(\frac{32 P_{49}}{9 N^{3}(1+N)^{3}(2+N)}\right. \\
& \left.-\frac{64(-5+N)(-1+2 N)}{N^{2}(1+N)^{2}} S_{1}-\frac{192(N-1)}{N(1+N)} S_{1}^{2}-\frac{128(N-1)}{N(1+N)} S_{2}\right) S_{-2}
\end{aligned}
$$




$$
\begin{aligned}
& -\frac{96(N-1)}{N(1+N)} S_{-2}^{2}+\left(-\frac{32\left(69-92 N+11 N^{3}\right)}{3 N^{2}(1+N)^{2}}-\frac{512(N-1)}{N(1+N)} S_{1}\right) S_{-3} \\
& -\frac{352(N-1)}{N(1+N)} S_{-4}-\frac{32(N-1)\left(24+11 N+11 N^{2}\right)}{3 N^{2}(1+N)^{2}} S_{2,1}-\frac{128(N-1)}{N(1+N)} S_{3,1} \\
& -\frac{64(-7+11 N)}{N^{2}(1+N)^{2}} S_{-2,1}+\frac{448(-1+N)}{N(1+N)} S_{-2,2}+\frac{512(-1+N)}{N(1+N)} S_{-3,1} \\
& \left.-\frac{768(-1+N)}{N(1+N)} S_{-2,1,1}+\frac{96(-1+N)\left(-8+3 N+3 N^{2}\right)}{N^{2}(1+N)^{2}} \zeta_{3}\right] \\
& +C_{F}^{2} T_{F} N_{F}\left[-\frac{8 P_{26}}{N^{3}(1+N)^{3}} S_{1}^{2}+\frac{8 P_{28}}{N^{3}(1+N)^{3}} S_{2}+\frac{P_{63}}{N^{4}(1+N)^{5}(2+N)}\right. \\
& +\left(-\frac{8 P_{53}}{N^{4}(1+N)^{4}}-\frac{8\left(-6+7 N+28 N^{2}+3 N^{3}\right)}{N^{2}(1+N)^{2}} S_{2}-\frac{704(N-1)}{3 N(1+N)} S_{3}\right. \\
& \left.+\frac{256(N-1)}{N(1+N)} S_{2,1}\right) S_{1}-\frac{8(N-1)\left(-10-9 N+3 N^{2}\right)}{3 N^{2}(1+N)^{2}} S_{1}^{3}-\frac{16(N-1)}{3 N(1+N)} S_{1}^{4} \\
& -\frac{48(N-1)}{N(1+N)} S_{2}^{2}-\frac{16(N-1)\left(-22+27 N+3 N^{2}\right)}{3 N^{2}(1+N)^{2}} S_{3}-\frac{160(N-1)}{N(1+N)} S_{4} \\
& +\left(\frac{64 P_{21}}{N^{2}(1+N)^{3}(2+N)}-\frac{256(N-1)}{N(1+N)^{2}} S_{1}-\frac{128(N-1)}{N(1+N)} S_{2}\right) S_{-2}-\frac{64(N-1)}{N(1+N)} S_{-2}^{2} \\
& +\left(-\frac{128(N-1)^{2}}{N^{2}(1+N)^{2}}-\frac{256(N-1)}{N(1+N)} S_{1}\right) S_{-3}-\frac{320(N-1)}{N(1+N)} S_{-4}-\frac{128(N-1)}{N^{2}(1+N)^{2}} S_{2,1} \\
& +\frac{64(N-1)}{N(1+N)} S_{3,1}+\frac{256(N-1)}{N(1+N)^{2}} S_{-2,1}+\frac{128(N-1)}{N(1+N)} S_{-2,2}+\frac{256(N-1)}{N(1+N)} S_{-3,1} \\
& \left.-\frac{192(N-1)}{N(1+N)} S_{2,1,1}+\frac{96(N-1)\left(-2+3 N+3 N^{2}\right)}{N^{2}(1+N)^{2}} \zeta_{3}\right], \\
& \Delta \gamma_{g q}^{(2)}=C_{F}^{2}\left[C _ { A } \left[\frac{4 P_{39}}{9 N^{3}(1+N)^{3}} S_{2}+\frac{P_{73}}{54(N-1) N^{5}(1+N)^{5}}+\left(-\frac{4 P_{58}}{27 N^{4}(1+N)^{4}}\right.\right.\right. \\
& -\frac{8\left(30+203 N+177 N^{2}+49 N^{3}\right)}{3 N^{2}(1+N)^{2}} S_{2}-\frac{640(2+N)}{3 N(1+N)} S_{3}+\frac{64(2+N)}{N(1+N)} S_{2,1} \\
& \left.+\frac{128(2+N)}{N(1+N)} S_{-2,1}+\frac{288(2+N)}{N(1+N)} \zeta_{3}\right) S_{1}+\left(\frac{4 P_{36}}{9 N^{3}(1+N)^{3}}-\frac{16(2+N)}{N(1+N)} S_{2}\right) S_{1}^{2} \\
& -\frac{8\left(6+85 N+132 N^{2}+50 N^{3}\right)}{9 N^{2}(1+N)^{2}} S_{1}^{3}+\frac{8(2+N)\left(102+65 N+29 N^{2}\right)}{9 N^{2}(1+N)^{2}} S_{3} \\
& +\frac{16(2+N)}{3 N(1+N)} S_{1}^{4}-\frac{32(2+N)}{N(1+N)} S_{4}+\left(-\frac{16 P_{44}}{(N-1) N^{3}(1+N)^{3}}+\frac{64(7+3 N)}{N(1+N)} S_{1}\right. \\
& \left.-\frac{96(2+N)}{N(1+N)} S_{1}^{2}\right) S_{-2}+\left(\frac{32(2+N)(4+3 N)}{N(1+N)^{2}}-\frac{64(2+N)}{N(1+N)} S_{1}\right) S_{-3}
\end{aligned}
$$




$$
\begin{aligned}
& +\frac{80(2+N)}{N(1+N)}\left[S_{-2}^{2}+S_{-4}\right]+\frac{16(2+N)\left(-6+11 N+11 N^{2}\right)}{3 N^{2}(1+N)^{2}} S_{2,1}+\frac{224(2+N)}{N(1+N)} S_{3,1} \\
& -\frac{32(2+N)(5+3 N)}{N(1+N)^{2}} S_{-2,1}+\frac{32(2+N)}{N(1+N)} S_{-2,2}-\frac{96(2+N)}{N(1+N)} S_{2,1,1} \\
& \left.-\frac{128(2+N)}{N(1+N)} S_{-2,1,1}-\frac{432(2+N) \zeta_{3}}{N(1+N)}\right]+T_{F} N_{F}\left[\frac{2 P_{71}}{27(N-1) N^{5}(1+N)^{5}}\right. \\
& +\left(\frac{32(2+N) P_{14}}{27 N^{3}(1+N)^{3}}+\frac{208(2+N)}{3 N(1+N)} S_{2}\right) S_{1}-\frac{16(2+N)\left(-3+16 N+37 N^{2}\right)}{9 N^{2}(1+N)^{2}} S_{1}^{2} \\
& +\frac{80(2+N)}{9 N(1+N)} S_{1}^{3}-\frac{16(2+N)\left(9+46 N+67 N^{2}\right)}{9 N^{2}(1+N)^{2}} S_{2}+\frac{256(2+N)}{9 N(1+N)} S_{3} \\
& \left.\left.+\frac{256}{(N-1) N^{2}(1+N)^{2}} S_{-2}-\frac{64(2+N)}{3 N(1+N)} S_{2,1}-\frac{128(2+N)}{N(1+N)} \zeta_{3}\right]\right] \\
& +C_{F}\left[T _ { F } ^ { 2 } N _ { F } ^ { 2 } \left[\frac{64(2+N)\left(3+7 N+N^{2}\right)}{9 N(1+N)^{3}}+\frac{64(2+N)(2+5 N)}{9 N(1+N)^{2}} S_{1}\right.\right. \\
& \left.-\frac{32(2+N)}{3 N(1+N)}\left[S_{1}^{2}+S_{2}\right]\right]+C_{A} T_{F} N_{F}\left[\frac{8 P_{57}}{27(N-1) N^{3}(1+N)^{4}}\right. \\
& +\left(-\frac{16 P_{37}}{27 N^{3}(1+N)^{3}}+\frac{80(2+N) S_{2}}{3 N(1+N)}\right) S_{1}+\frac{16\left(18+116 N+129 N^{2}+43 N^{3}\right)}{9 N^{2}(1+N)^{2}} S_{1}^{2} \\
& -\frac{80(2+N)}{9 N(1+N)} S_{1}^{3}+\frac{16\left(-2+16 N+9 N^{2}+N^{3}\right)}{3 N^{2}(1+N)^{2}} S_{2}+\frac{512(2+N)}{9 N(1+N)} S_{3} \\
& +\left(-\frac{64 P_{7}}{3(-1+N) N^{2}(1+N)^{2}}+\frac{256(2+N) S_{1}}{3 N(1+N)}\right) S_{-2}+\frac{128(2+N)}{3 N(1+N)}\left[S_{-3}-S_{-2,1}\right] \\
& \left.+\frac{128(2+N)}{N(1+N)} \zeta_{3}\right]+C_{A}^{2}\left[\frac{2 P_{35}}{3 N^{3}(1+N)^{3}} S_{2}-\frac{4 P_{72}}{27(N-1) N^{5}(1+N)^{5}}\right. \\
& +\left(\frac{4 P_{62}}{27(N-1) N^{4}(1+N)^{4}}-\frac{4\left(120+158 N+141 N^{2}+55 N^{3}\right)}{3 N^{2}(1+N)^{2}} S_{2}+\frac{128(2+N)}{3 N(1+N)} S_{3}\right. \\
& \left.+\frac{128(2+N)}{N(1+N)} S_{-2,1}-\frac{96(2+N)}{N(1+N)} \zeta_{3}\right) S_{1}+\left(-\frac{2 P_{38}}{9 N^{3}(1+N)^{3}}+\frac{48(2+N)}{N(1+N)} S_{2}\right) S_{1}^{2} \\
& +\frac{4\left(24+158 N+165 N^{2}+55 N^{3}\right)}{9 N^{2}(1+N)^{2}} S_{1}^{3}-\frac{8(2+N)}{3 N(1+N)} S_{1}^{4}-\frac{40(2+N)}{N(1+N)} S_{2}^{2} \\
& -\frac{8\left(-186+295 N+528 N^{2}+176 N^{3}\right)}{9 N^{2}(1+N)^{2}} S_{3}-\frac{16(2+N)}{N(1+N)} S_{4} \\
& +\left(-\frac{32 P_{12}}{3(N-1) N^{2}(1+N)^{2}} S_{1}+\frac{16 P_{47}}{3(N-1) N^{3}(1+N)^{3}}+\frac{96(2+N)}{N(1+N)} S_{1}^{2}\right. \\
& \left.-\frac{64(2+N)}{N(1+N)} S_{2}\right) S_{-2}-\frac{16(2+N)}{N(1+N)} S_{-2}^{2}+\left(-\frac{16\left(-126-13 N+66 N^{2}+22 N^{3}\right)}{3 N^{2}(1+N)^{2}}\right.
\end{aligned}
$$




$$
\begin{aligned}
& \left.-\frac{192(2+N)}{N(1+N)} S_{1}\right) S_{-3}-\frac{176(2+N)}{N(1+N)} S_{-4}+\frac{32\left(-30+13 N+33 N^{2}+11 N^{3}\right)}{3 N^{2}(1+N)^{2}} S_{-2,1} \\
& -\frac{64(2+N)}{N(1+N)} S_{3,1}+\frac{224(2+N)}{N(1+N)} S_{-2,2}+\frac{256(2+N)}{N(1+N)} S_{-3,1}-\frac{384(2+N)}{N(1+N)} S_{-2,1,1} \\
& \left.\left.+\frac{144(2+N)}{N(1+N)} \zeta_{3}\right]\right]+C_{F}^{3}\left[-\frac{2(2+N) P_{19}}{N^{3}(1+N)^{3}} S_{2}+\frac{P_{66}}{2(N-1) N^{5}(1+N)^{5}}\right. \\
& +\left(-\frac{4(2+N) P_{43}}{N^{4}(1+N)^{4}}+\frac{4(2+N)\left(-2+19 N+39 N^{2}\right)}{N^{2}(1+N)^{2}} S_{2}+\frac{128(2+N)}{3 N(1+N)} S_{3}\right. \\
& \left.-\frac{64(2+N)}{N(1+N)} S_{2,1}-\frac{192(2+N)}{N(1+N)} \zeta_{3}\right) S_{1}+\left(\frac{2(2+N) P_{3}}{N^{3}(1+N)^{3}}-\frac{32(2+N)}{N(1+N)} S_{2}\right) S_{1}^{2} \\
& +\frac{4(2+N)\left(-2+3 N+15 N^{2}\right)}{3 N^{2}(1+N)^{2}} S_{1}^{3}-\frac{8(2+N)}{3 N(1+N)} S_{1}^{4}-\frac{24(2+N)}{N(1+N)} S_{2}^{2} \\
& +\frac{64(2+N)\left(-1+3 N^{2}\right)}{3 N^{2}(1+N)^{2}} S_{3}-\frac{48(2+N)}{N(1+N)} S_{4}+\left(\frac{32 P_{22}}{(N-1) N^{2}(1+N)^{3}}\right. \\
& \left.-\frac{128(2+N)}{N(1+N)^{2}} S_{1}-\frac{64(2+N)}{N(1+N)} S_{2}\right) S_{-2}-\frac{16(2+N)\left(-2+3 N+3 N^{2}\right)}{N^{2}(1+N)^{2}} S_{2,1} \\
& -\frac{96(2+N)}{N(1+N)} S_{-2}^{2}+\left(-\frac{64(N-1)(2+N)}{N^{2}(1+N)^{2}}-\frac{128(2+N)}{N(1+N)} S_{1}\right) S_{-3} \\
& -\frac{160(2+N)}{N(1+N)}\left[S_{-4}+S_{3,1}\right]+\frac{128(2+N)}{N(1+N)^{2}}\left[S_{-2,1}+(1+N) S_{-3,1}\right] \\
& \left.+\frac{64(2+N)}{N(1+N)} S_{-2,2}+\frac{96(2+N)}{N(1+N)} S_{2,1,1}+\frac{288(2+N) \zeta_{3}}{N(1+N)}\right] \text {, } \\
& \Delta \gamma_{g g}^{(2)}=C_{A} T_{F}^{2} N_{F}^{2}\left[-\frac{16 P_{8}}{27 N^{2}(1+N)^{2}} S_{1}-\frac{4 P_{48}}{27 N^{3}(1+N)^{3}}\right]+C_{F}\left[T _ { F } ^ { 2 } N _ { F } ^ { 2 } \left[-\frac{8 P_{59}}{27 N^{4}(1+N)^{4}}\right.\right. \\
& +\frac{64(N-1)(2+N)\left(-6-8 N+N^{2}\right)}{9 N^{3}(1+N)^{3}} S_{1}+\frac{32(N-1)(2+N)}{3 N^{2}(1+N)^{2}} S_{1}^{2} \\
& \left.-\frac{32(N-1)(2+N)}{N^{2}(1+N)^{2}} S_{2}\right]+C_{A} T_{F} N_{F}\left[\frac{8 P_{6}}{N^{3}(1+N)^{3}} S_{2}-\frac{8 P_{9}}{3 N^{3}(1+N)^{3}} S_{1}^{2}\right. \\
& +\frac{2 P_{77}}{27(N-1) N^{5}(1+N)^{5}(2+N)}+\left(-\frac{8 P_{67}}{9(-1+N) N^{4}(1+N)^{4}(2+N)}\right. \\
& \left.-\frac{32(N-1)(2+N)}{N^{2}(1+N)^{2}} S_{2}+128 \zeta_{3}\right) S_{1}+\frac{32(N-1)(2+N)}{3 N^{2}(1+N)^{2}} S_{1}^{3}-\frac{32\left(34+N+N^{2}\right)}{3 N^{2}(1+N)^{2}} \\
& \times S_{3}+\left(\frac{128 P_{2}}{(N-1) N^{2}(1+N)^{2}(2+N)} S_{1}-\frac{32 P_{23}}{(N-1) N^{2}(1+N)^{3}(2+N)}\right) S_{-2} \\
& -\frac{192\left(4-N-N^{2}\right)}{N^{2}(1+N)^{2}} S_{-3}+\frac{64(N-1)(2+N)}{N^{2}(1+N)^{2}} S_{2,1}-\frac{128\left(-8+N+N^{2}\right)}{N^{2}(1+N)^{2}} S_{-2,1}
\end{aligned}
$$




$$
\begin{aligned}
& \left.\left.-\frac{64(-3+N)(4+N)}{N^{2}(1+N)^{2}} \zeta_{3}\right]\right]+C_{A}^{3}\left[\frac{64 P_{16}}{9 N^{2}(1+N)^{2}} S_{-2,1}-\frac{32 P_{18}}{9 N^{2}(1+N)^{2}} S_{3}\right. \\
& +\frac{P_{74}}{27(N-1) N^{5}(1+N)^{5}(2+N)}+\left(\frac{4 P_{69}}{9(N-1) N^{4}(1+N)^{4}(2+N)}\right. \\
& -\frac{64 P_{17}}{9 N^{2}(1+N)^{2}} S_{2}+128 S_{2}^{2}+\frac{16\left(-96+11 N+11 N^{2}\right)}{3 N(1+N)} S_{3}+192 S_{4} \\
& \left.+\frac{1024}{N(1+N)} S_{-2,1}-640 S_{-2,2}-768 S_{-3,1}+1024 S_{-2,1,1}\right) S_{1} \\
& +\left(-\frac{256\left(1+3 N+3 N^{2}\right)}{N^{3}(1+N)^{3}}+128 S_{3}-256 S_{-2,1}\right) S_{1}^{2}+\left(-\frac{16 P_{41}}{9 N^{3}(1+N)^{3}}\right. \\
& \left.+64 S_{3}+640 S_{-2,1}\right) S_{2}-\frac{256}{N(1+N)} S_{2}^{2}-\frac{384}{N(1+N)} S_{4}+64 S_{5} \\
& +\left(\frac{32 P_{52}}{9(N-1) N^{3}(1+N)^{3}(2+N)}+\left(-\frac{64 P_{32}}{9(-1+N) N(1+N)^{2}(2+N)}+256 S_{2}\right)\right. \\
& \left.\times S_{1}-\frac{512}{N(1+N)} S_{2}+128 S_{3}-768 S_{2,1}\right) S_{-2}+\left(-\frac{16\left(24+11 N+11 N^{2}\right)}{3 N(1+N)}\right. \\
& \left.+64 S_{1}\right) S_{-2}^{2}+\left(-\frac{32 P_{15}}{9 N^{2}(1+N)^{2}}-\frac{1536}{N(1+N)} S_{1}+384 S_{1}^{2}-320 S_{2}\right) S_{-3} \\
& +\left(-\frac{1024}{N(1+N)}+512 S_{1}\right) S_{-4}-192 S_{-5}-384 S_{2,-3}+\frac{1280}{N(1+N)} S_{-2,2} \\
& +384 S_{-2,3}+\frac{1536}{N(1+N)} S_{-3,1}-384 S_{-4,1}+768 S_{2,1,-2}-\frac{2048}{N(1+N)} S_{-2,1,1} \\
& \left.+768\left[S_{-2,2,1}+S_{-3,1,1}\right]-1536 S_{-2,1,1,1}\right] \\
& +C_{F}^{2} T_{F} N_{F}\left[-\frac{4 P_{75}}{(N-1) N^{5}(1+N)^{5}(2+N)}+\left(\frac{32(N-1)(2+N) S_{2}}{N^{2}(1+N)^{2}}\right.\right. \\
& \left.-\frac{16 P_{42}}{N^{4}(1+N)^{4}}\right) S_{1}+\frac{8(N-1)(2+N)\left(2+3 N+3 N^{2}\right)}{N^{3}(1+N)^{3}} S_{1}^{2}-\frac{32(N-1)(2+N)}{3 N^{2}(1+N)^{2}} \\
& \times S_{1}^{3}-\frac{8(2+N)\left(2-11 N-16 N^{2}+9 N^{3}\right)}{N^{3}(1+N)^{3}} S_{2}+\frac{32\left(10+7 N+7 N^{2}\right)}{3 N^{2}(1+N)^{2}} S_{3} \\
& +\left(-\frac{64\left(10+N+N^{2}\right)}{(N-1) N(1+N)(2+N)}+\frac{512}{N^{2}(1+N)^{2}} S_{1}\right) S_{-2}+\frac{256}{N^{2}(1+N)^{2}} S_{-3} \\
& \left.-\frac{64(N-1)(2+N)}{N^{2}(1+N)^{2}} S_{2,1}-\frac{512}{N^{2}(1+N)^{2}} S_{-2,1}+\frac{192\left(-2-N-N^{2}\right)}{N^{2}(1+N)^{2}} \zeta_{3}\right] \\
& +C_{A}^{2} T_{F} N_{F}\left[\frac{32 P_{4}}{9 N^{2}(1+N)^{2}} S_{2}+\frac{32 P_{11}}{9 N^{2}(1+N)^{2}} S_{-3}-\frac{64 P_{11}}{9 N^{2}(1+N)^{2}} S_{-2,1}\right.
\end{aligned}
$$




$$
\begin{aligned}
& +\frac{16 P_{13}}{9 N^{2}(1+N)^{2}} S_{3}+\frac{2 P_{76}}{27(N-1) N^{5}(1+N)^{5}(2+N)}+\left(\frac{1280}{9} S_{2}-\frac{64}{3} S_{3}\right. \\
& \left.-\frac{8 P_{68}}{27(-1+N) N^{4}(1+N)^{4}(2+N)}-128 \zeta_{3}\right) S_{1}+\frac{64}{3} S_{-2}^{2} \\
& +\left(\frac{64 P_{45}}{9(N-1) N^{2}(1+N)^{2}(2+N)} S_{1}-\frac{32 P_{50}}{9(N-1) N^{3}(1+N)^{3}(2+N)}\right) S_{-2} \\
& \left.+\frac{128\left(-3+2 N+2 N^{2}\right)}{N^{2}(1+N)^{2}} \zeta_{3}\right],
\end{aligned}
$$

with the polynomials

$$
\begin{aligned}
& P_{1}=N^{4}-2 N^{3}-4 N^{2}+15 N+2, \\
& P_{2}=N^{4}+2 N^{3}-5 N^{2}-6 N+16 \text {, } \\
& P_{3}=N^{4}+44 N^{3}+45 N^{2}+38 N+12, \\
& P_{4}=3 N^{4}+6 N^{3}-89 N^{2}-92 N+12, \\
& P_{5}=3 N^{4}+6 N^{3}+16 N^{2}+13 N-3 \text {, } \\
& P_{6}=3 N^{4}+18 N^{3}+17 N^{2}-46 N-28 \text {, } \\
& P_{7}=5 N^{4}+9 N^{3}-4 N^{2}-4 N+6 \text {, } \\
& P_{8}=8 N^{4}+16 N^{3}-19 N^{2}-27 N+48 \text {, } \\
& P_{9}=11 N^{4}+22 N^{3}+13 N^{2}+2 N-12, \\
& P_{10}=11 N^{4}+34 N^{3}+N^{2}-70 N-12, \\
& P_{11}=20 N^{4}+40 N^{3}+11 N^{2}-9 N+54, \\
& P_{12}=22 N^{4}+50 N^{3}+5 N^{2}-47 N+6, \\
& P_{13}=40 N^{4}+80 N^{3}+73 N^{2}+33 N+54, \\
& P_{14}=62 N^{4}-17 N^{3}-76 N^{2}-69 N-18 \text {, } \\
& P_{15}=67 N^{4}+134 N^{3}+49 N^{2}+54 N-360, \\
& P_{16}=67 N^{4}+134 N^{3}+49 N^{2}+54 N-72 \text {, } \\
& P_{17}=67 N^{4}+134 N^{3}+67 N^{2}+144 N+72, \\
& P_{18}=67 N^{4}+134 N^{3}+109 N^{2}+114 N-126, \\
& P_{19}=95 N^{4}+148 N^{3}+35 N^{2}-38 N-12 \text {, } \\
& P_{20}=N^{5}+N^{4}-4 N^{3}+3 N^{2}-7 N-2, \\
& P_{21}=2 N^{5}+6 N^{4}-N^{3}-8 N^{2}+15 N+10, \\
& P_{22}=2 N^{5}+6 N^{4}+5 N^{3}+4 N^{2}+9 N-2, \\
& P_{23}=3 N^{5}+5 N^{4}-33 N^{3}-45 N^{2}+6 N-16, \\
& P_{24}=3 N^{5}+14 N^{4}+21 N^{3}+20 N^{2}+10 N+4, \\
& P_{25}=8 N^{5}-31 N^{4}+205 N^{3}-59 N^{2}-447 N-108 \text {, } \\
& P_{26}=14 N^{5}+15 N^{4}-19 N^{3}-13 N^{2}-25 N-20 \text {, }
\end{aligned}
$$




$$
\begin{aligned}
& P_{27}=21 N^{5}+9 N^{4}+13 N^{3}-13 N^{2}-22 N-12, \\
& P_{28}=26 N^{5}+41 N^{4}-21 N^{3}+21 N^{2}+9 N-12 \text {, } \\
& P_{29}=36 N^{5}-55 N^{4}-243 N^{3}-75 N^{2}-163 N-108, \\
& P_{30}=58 N^{5}+7 N^{4}+59 N^{3}+50 N^{2}+3 N-9, \\
& P_{31}=67 N^{5}+49 N^{4}-52 N^{3}+164 N^{2}-90 N-72, \\
& P_{32}=67 N^{5}+201 N^{4}+67 N^{3}-57 N^{2}+109 N-189, \\
& P_{33}=76 N^{5}+271 N^{4}+254 N^{3}+41 N^{2}+72 N+36, \\
& P_{34}=85 N^{5}+151 N^{4}-40 N^{3}+164 N^{2}-306 N-72, \\
& P_{35}=171 N^{5}+552 N^{4}+343 N^{3}+246 N^{2}+1052 N+480, \\
& P_{36}=305 N^{5}+989 N^{4}+907 N^{3}-5 N^{2}+60 N+36, \\
& P_{37}=418 N^{5}+1525 N^{4}+1763 N^{3}+650 N^{2}+444 N+144 \text {, } \\
& P_{38}=631 N^{5}+2524 N^{4}+3743 N^{3}+3398 N^{2}+2844 N+864, \\
& P_{39}=725 N^{5}+2831 N^{4}+3481 N^{3}+1699 N^{2}+1044 N+324, \\
& P_{40}=N^{6}+3 N^{5}+5 N^{4}+N^{3}-8 N^{2}+2 N+4, \\
& P_{41}=3 N^{6}+9 N^{5}-584 N^{4}-1183 N^{3}-275 N^{2}-834 N-432 \text {, } \\
& P_{42}=5 N^{6}+23 N^{5}+11 N^{4}-39 N^{3}-20 N^{2}+16 N+8, \\
& P_{43}=9 N^{6}+56 N^{5}+87 N^{4}+54 N^{3}-52 N^{2}-50 N-12, \\
& P_{44}=14 N^{6}+39 N^{5}+14 N^{4}-19 N^{3}+6 N^{2}+14 N+4, \\
& P_{45}=20 N^{6}+60 N^{5}+11 N^{4}-78 N^{3}-13 N^{2}+36 N-108, \\
& P_{46}=48 N^{6}+144 N^{5}+469 N^{4}+698 N^{3}+7 N^{2}+258 N+144 \text {, } \\
& P_{47}=76 N^{6}+217 N^{5}+25 N^{4}-181 N^{3}+106 N^{2}-21 N-6 \text {, } \\
& P_{48}=87 N^{6}+261 N^{5}+249 N^{4}+63 N^{3}-76 N^{2}-64 N-96 \text {, } \\
& P_{49}=94 N^{6}+315 N^{5}+145 N^{4}-63 N^{3}+148 N^{2}-441 N-18, \\
& P_{50}=95 N^{6}+285 N^{5}+92 N^{4}-291 N^{3}-97 N^{2}+96 N-36 \text {, } \\
& P_{51}=160 N^{6}+438 N^{5}+364 N^{4}+330 N^{3}+529 N^{2}+321 N+18, \\
& P_{52}=325 N^{6}+975 N^{5}+85 N^{4}-879 N^{3}+598 N^{2}-240 N-72 \text {, } \\
& P_{53}=17 N^{7}+9 N^{6}-95 N^{5}-19 N^{4}+76 N^{3}+22 N^{2}+26 N+28, \\
& P_{54}=24 N^{7}+33 N^{6}+13 N^{5}-28 N^{4}-31 N^{3}-33 N^{2}-26 N-8 \text {, } \\
& P_{55}=165 N^{7}+330 N^{6}-491 N^{5}-365 N^{4}-136 N^{3}-445 N^{2}-18 N+144 \text {, } \\
& P_{56}=475 N^{7}+833 N^{6}+1527 N^{5}+2905 N^{4}-1342 N^{3}+5562 N^{2}+3834 N+486 \text {, } \\
& P_{57}=537 N^{7}+1200 N^{6}-1013 N^{5}-2085 N^{4}+1720 N^{3}-855 N^{2}-2468 N-492 \text {, } \\
& P_{58}=1199 N^{7}+3523 N^{6}+681 N^{5}-5953 N^{4}-4214 N^{3}-4800 N^{2}-3168 N-864 \text {, } \\
& P_{59}=33 N^{8}+132 N^{7}+70 N^{6}-612 N^{5}-839 N^{4}+480 N^{3}+712 N^{2}+408 N+144 \text {, } \\
& P_{60}=476 N^{8}+2297 N^{7}+2018 N^{6}-4915 N^{5}-7324 N^{4}+242 N^{3}-1218 N^{2} \\
& -2700 N-864 \text {, }
\end{aligned}
$$




$$
\begin{aligned}
& P_{61}=914 N^{8}+3005 N^{7}+3368 N^{6}+4349 N^{5}+5183 N^{4}+548 N^{3}+1101 N^{2} \\
& +936 N+324 \text {, } \\
& P_{62}=2078 N^{8}+8225 N^{7}+10475 N^{6}-1921 N^{5}-10729 N^{4}-2560 N^{3}-5658 N^{2}-7578 N \\
& -2700 \text {, } \\
& P_{63}=-5 N^{9}-25 N^{8}+228 N^{7}+926 N^{6}-201 N^{5}-2377 N^{4}+626 N^{3}+2788 N^{2}+2168 N \\
& +480 \text {, } \\
& P_{64}=99 N^{9}+297 N^{8}-982 N^{7}-662 N^{6}+1035 N^{5}-3079 N^{4}+3448 N^{3}+2868 N^{2} \\
& -2448 N-1728 \text {, } \\
& P_{65}=-1251 N^{10}-6255 N^{9}-10972 N^{8}-17422 N^{7}-1423 N^{6}+111905 N^{5}+149894 N^{4} \\
& -2116 N^{3}-37752 N^{2}+12384 N+6912, \\
& P_{66}=-115 N^{10}-383 N^{9}+356 N^{8}+2762 N^{7}+3001 N^{6}-471 N^{5}-882 N^{4}+1068 N^{3} \\
& +1352 N^{2}-288 N-256 \text {, } \\
& P_{67}=165 N^{10}+825 N^{9}+1102 N^{8}-578 N^{7}-1939 N^{6}-239 N^{5}+1184 N^{4}+448 N^{3} \\
& -2456 N^{2}-2256 N-864 \text {, } \\
& P_{68}=418 N^{10}+2090 N^{9}+3857 N^{8}+5096 N^{7}+6254 N^{6}-808 N^{5}-10295 N^{4}-5622 N^{3} \\
& +2898 N^{2}+2376 N+648 \\
& P_{69}=735 N^{10}+3675 N^{9}+6060 N^{8}+6934 N^{7}+11743 N^{6}-41 N^{5}-18290 N^{4}-920 N^{3} \\
& -8168 N^{2}-10656 N-3744 \text {, } \\
& P_{70}=741 N^{10}+3705 N^{9}+2650 N^{8}-8780 N^{7}-12083 N^{6}-13127 N^{5}-15536 N^{4}+3586 N^{3} \\
& -16128 N^{2}-11916 N-3240 \text {, } \\
& P_{71}=1065 N^{10}+6693 N^{9}+14084 N^{8}+10058 N^{7}-3475 N^{6}-11707 N^{5}+446 N^{4}+17132 N^{3} \\
& +3432 N^{2}-6624 N-3456, \\
& P_{72}=3321 N^{10}+13584 N^{9}+9571 N^{8}-17159 N^{7}-7838 N^{6}+5281 N^{5}-20690 N^{4}-842 N^{3} \\
& -5208 N^{2}-7884 N-3240, \\
& P_{73}=18579 N^{10}+68775 N^{9}+3212 N^{8}-235282 N^{7}-220465 N^{6}+54263 N^{5}+91994 N^{4} \\
& -48748 N^{3}-24648 N^{2}+29664 N+13824 \text {, } \\
& P_{74}=-2133 N^{12}-12798 N^{11}-54337 N^{10}-153794 N^{9}-137083 N^{8}+105398 N^{7} \\
& +31109 N^{6}-29734 N^{5}+318684 N^{4}-18512 N^{3}+82224 N^{2}+126720 N+48384 \text {, (4.89) } \\
& P_{75}=N^{12}+6 N^{11}-27 N^{10}-186 N^{9}-197 N^{8}+310 N^{7}+899 N^{6}+1198 N^{5}+1020 N^{4} \\
& +112 N^{3}-192 N^{2}+64 N+64, \\
& P_{76}=699 N^{12}+4194 N^{11}+16447 N^{10}+43214 N^{9}+42657 N^{8}-19098 N^{7}-36963 N^{6} \\
& -11670 N^{5}-45064 N^{4}-39392 N^{3}+7536 N^{2}+8064 N+1728, \\
& P_{77}=723 N^{12}+4338 N^{11}+12623 N^{10}+17230 N^{9}-8583 N^{8}-30018 N^{7}+47709 N^{6} \\
& +75738 N^{5}+8776 N^{4}+67208 N^{3}+4416 N^{2}-41184 N-20736 .
\end{aligned}
$$


We mention the moment-relation

$$
\Delta \gamma_{g g}^{(2)}(N=1)=-2 \beta_{2}
$$

with $[73-84]$

$$
\beta_{2}=\frac{2857}{54} C_{A}^{3}+T_{F} N_{F}\left[-\frac{1415}{27} C_{A}^{2}-\frac{205}{9} C_{A} C_{F}+2 C_{F}^{2}\right]+\left(T_{F} N_{F}\right)^{2}\left[\frac{158}{27} C_{A}+\frac{44}{9} C_{F}\right] .
$$

The so-called supersymmetric relation obtained by setting $C_{F}=C_{A}=2 T_{F} N_{F}$ is broken using dimensional regularization from $O\left(a_{s}^{2}\right)$ onward, while the relation

$$
\Delta \gamma_{q q}^{(k)}+\Delta \gamma_{g q}^{(k)}-\Delta \gamma_{q g}^{(k)}-\Delta \gamma_{g g}^{(k)}=0
$$

also holds at $k=1$ using dimensional reduction [26] in this limit.

Furthermore, one has

$$
\Delta \gamma_{q g}^{(k)}(N=1)=0, \text { for } k=0,1,2
$$

The expansions have been performed using the HarmonicSums command HarmonicSumsSeries since (4.13), (4.15) contain evanescent poles at $N=1$. More generally, also the first moment of the gluonic Wilson coefficient, related to (4.96), for the structure function $g_{1}\left(x, Q^{2}\right)$ both for the massless $[72,157]$ and the massive case in the asymptotic representation to two-loop order [158] vanishes. This is known at one-loop order even for general kinematics [159-161].

The splitting functions in $z$-space are obtained by a Mellin inversion, cf. (2.35), and are given in computer readable form in the supplementary material.

\section{Comparison to the literature}

We confirm the results for the singlet anomalous dimensions calculated in [30], where the on-shell forward Compton amplitude has been used for the computation. The contributions $\propto T_{F}$ have already been calculated independently as a by-product of the massive on-shell operator matrix elements in ref. [31], to which we also agree. The comparison to the large $N_{F}$ expansions of refs. $[162,163]$ has been given in our previous paper [31] already, where all these terms are covered.

Let us finally consider the small $z \operatorname{limit}^{5}$ of the present results and compare to the predictions given in $[165,166]$. In Mellin- $N$ space these terms are given by the most singular contribution expanding around $N=0$. One obtains at one- and two-loop order

$$
\begin{aligned}
\Delta \gamma^{(0), N \rightarrow 0} & =-\frac{4}{N}\left(\begin{array}{cc}
C_{F} & -2 T_{F} N_{F} \\
2 C_{F} & 4 C_{A}
\end{array}\right) \\
\Delta \gamma^{(1), N \rightarrow 0} & =-\frac{8}{N^{3}}\left(\begin{array}{cc}
C_{F}\left(2 C_{A}-3 C_{F}-4 T_{F} N_{F}\right) & 2 C_{F}\left(2 C_{A}+C_{F}\right) \\
-2 C_{F}\left(2 C_{A}+C_{F}\right) & 4\left(2 C_{A}^{2}-C_{F} T_{F} N_{F}\right)
\end{array}\right)
\end{aligned}
$$

which agree with the evolution kernels given in refs. [165, 166].

\footnotetext{
${ }^{5}$ For a review on the small $z$ predictions of the different evolution kernels see [164].
} 
At three-loop order we have in the M-scheme

$$
\begin{aligned}
& \Delta \gamma^{(2), N \rightarrow 0}=-\frac{16}{N^{5}} \\
& \times\left(\begin{array}{cc}
C_{F}\left(3 C_{A}^{2}-12 C_{A} C_{F}+10 C_{F}^{2}+T_{F} N_{F}\left(12 C_{A}+16 C_{F}\right)\right) & 2 T_{F} N_{F}\left(15 C_{A}^{2}+4 C_{A} C_{F}-8 C_{F} T_{F} N_{F}\right) \\
-2 C_{F}\left(15 C_{A}^{2}+8 C_{A} C_{F}-4 C_{F}^{2}-8 C_{F} T_{F} N_{F}\right) & -4\left(14 C_{A}^{3}+T_{F} N_{F}\left(C_{A}^{2}-12 C_{A} C_{F}-2 C_{F}^{2}\right)\right)
\end{array}\right)
\end{aligned}
$$

and find a deviation both in case of $\Delta \gamma_{q g}^{(2)}$ and $\Delta \gamma_{g q}^{(2)}$, already noticed in [167]. The relative deviation amounts to $\sim \pm 3.2 \%$, with the difference of the expression in the M-scheme and the result obtained for the infrared evolution equation (IEE), $\delta \Delta \gamma_{i j}^{(2), N \rightarrow 0}=\Delta \gamma_{i j}^{(2), N \rightarrow 0, \mathrm{M}}$

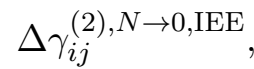

$$
\begin{aligned}
\delta \Delta \gamma_{q g}^{(2), N \rightarrow 0} & =\frac{48}{N^{5}} C_{F} T_{F} N_{F}\left(C_{A}-C_{F}\right), \\
\delta \Delta \gamma_{g q}^{(2), N \rightarrow 0} & =\frac{64}{N^{5}} C_{F}^{2}\left(C_{A}-C_{F}\right) .
\end{aligned}
$$

One may consider a different theory but QCD by setting $C_{A}=C_{F}$. In this case the prediction $[165,166]$ for the evolution kernels agrees with the perturbative calculation of the anomalous dimensions.

It has been the group of J. Kodaira [168], who also considered the effective Wilson coefficient in the non-singlet case ref. [169] ${ }^{6}$ finding that these contributions are suppressed by a further power in $N$. This also applies to the effective Wilson coefficients in the nonsinglet case and therefore the evolution kernels of $[169,170]$ agree. $^{7}$ By considering the expansion of the function to be interpreted as a matrix formulation for the effective Wilson coefficient in [165],

$$
\frac{N}{\mathbf{1} N-\frac{1}{8 \pi^{2}} \mathbf{F}_{0}}=\mathbf{1}+\sum_{k=1}^{\infty}\left(\frac{a_{s}}{N^{2}}\right)^{k} \mathbf{F}_{0, k} .
$$

The expansion coefficients $\mathbf{F}_{0, k}$ are $2 \times 2$ matrices only depending on color factors. Therefore, in the representation of [165], the Wilson coefficients are suppressed by one power in $N$, like in the non-singlet case, cf. $[168,169]$. To perform a full comparison one has to form two scheme invariant quantities. This is not really possible in pure polarized QCD, since there is only one structure function $g_{1}\left(x, Q^{2}\right)$ and also considering the Wilson coefficients $[72,157] .{ }^{8}$ In $[30]$ scheme invariant polarized evolution kernels for the contributions to the structure function $g_{1}$ and additional fictitious gravitational contributions in the gluonic channels have been formed for which the prediction in $[165,166]$ hold.

In data analyses or the phenomenological description of the polarized structure functions the consideration of only the leading small $z$ terms in the evolution kernels is numerically not sufficient. Subleading terms dominate over the leading order effects, cf. [164, 166, 171].

\footnotetext{
${ }^{6}$ In using infrared evolution equations the authors of refs. [165, 169, 170] do not specify the factorization scheme, which complicates comparisons with calculations performed e.g. in the M-scheme.

${ }^{7}$ After correcting ref. [170] in [171].

${ }^{8}$ At the level of twist-2 the structure function $g_{2}\left(x, Q^{2}\right)$ is not an independent quantity, but related by the Wandzura-Wilczek relation [172] to the structure function $g_{1}\left(x, Q^{2}\right)$. One might consider the physical pair $\left\{g_{1}\left(x, Q^{2}\right), \partial g_{1}\left(x, Q^{2}\right) / \partial \ln \left(Q^{2}\right)\right\}$, cf. [69, 70]. However, the so-called leading powers in $N$ are here not of the same order.
} 


\section{Conclusions}

We have calculated the polarized three-loop singlet anomalous dimensions $\Delta \gamma_{q q}^{(2), \mathrm{PS}}, \Delta \gamma_{q g}^{(2)}$, $\Delta \gamma_{g q}^{(2)}, \Delta \gamma_{g g}^{(2)}$, and the non-singlet anomalous dimension $\Delta \gamma_{q q}^{(2), \mathrm{s}, \mathrm{NS}}$ in Quantum Chromodynamics and agree with the results of refs. [30, 31, 50]. The singlet anomalous dimensions have been calculated by using the method of massless off-shell OMEs, which has been applied for this purpose for the first time. The calculation has been fully automated referring to the Larin scheme, performing a finite transformation to the M-scheme for the final results. Both schemes are valid to describe the scaling violations of the polarized structure functions, however, with a different outcome for the polarized parton distribution functions, which are scheme-dependent quantities. Comparing to predictions of the leading small $z$ behaviour one finds deviations for the off-diagonal elements at three-loop order, from which one concludes that the calculation in ref. [165] is not in the M-scheme starting with three-loop order. To obtain the complete picture, one has to consider also the associated behaviour of the Wilson coefficients and to form scheme-invariant quantities. We also mention that partial checks on the polarized anomalous dimensions are possible from the pole terms of the single- and two-mass massive OMEs to three loop order, cf. [173-180]. Both the anomalous dimension and splitting functions are given in computer readable form in the supplementary material file ANOM3pol.m attached to this paper.

\section{Acknowledgments}

We would like to thank J. Ablinger and A. Vogt for a discussion. This project has received funding from the European Union's Horizon 2020 research and innovation programme under the Marie Skłodowska-Curie grant agreement No. 764850, SAGEX and from the Austrian Science Fund (FWF) grants SFB F50 (F5009-N15) and P33530.

\section{A A new polarized gluonic Feynman rule}

The gluonic OME $\Delta A_{g g}$ requires a new Feynman rule to calculate the anomalous dimensions in the massless case, containing a local operator with five external gluon lines, extending the setting given in refs. $[31,52]$. The operator insertion reads in the polarized case

$$
\begin{aligned}
& p_{1, \nu_{1}, c_{1}}{ }_{p_{2}, \nu_{2}, c_{2}}{ }^{2}{ }_{p_{4}, \nu_{4}, c_{4}}=-i g^{3} \frac{1-(-1)^{N}}{2}\left(f^{c_{1} c_{2} c_{x}} f^{c_{3} c_{x} c_{y}} f^{c_{4} c_{5} c_{y}} O_{\nu_{1} \nu_{2} \nu_{3} \nu_{4} \nu_{5}}\left(p_{1}, p_{2}, p_{3}, p_{4}, p_{5}\right)\right. \\
& +f^{c_{1} c_{2} c_{x}} f^{c_{4} c_{x} c_{y}} f^{c_{3} c_{5} c_{y}} O_{\nu_{1} \nu_{2} \nu_{4} \nu_{3} \nu_{5}}\left(p_{1}, p_{2}, p_{4}, p_{3}, p_{5}\right)+f^{c_{1} c_{2} c_{x}} f^{c_{5} c_{x} c_{y}} f^{c_{3} c_{4} c_{y}} O_{\nu_{1} \nu_{2} \nu_{5} \nu_{3} \nu_{4}}\left(p_{1}, p_{2}, p_{5}, p_{3}, p_{4}\right) \\
& +f^{c_{1} c_{3} c_{x}} f^{c_{2} c_{x} c_{y}} f^{c_{4} c_{5} c_{y}} O_{\nu_{1} \nu_{3} \nu_{2} \nu_{4} \nu_{5}}\left(p_{1}, p_{3}, p_{2}, p_{4}, p_{5}\right)+f^{c_{1} c_{3} c_{x}} f^{c_{4} c_{x} c_{y}} f^{c_{2} c_{5} c_{y}} O_{\nu_{1} \nu_{3} \nu_{4} \nu_{2} \nu_{5}}\left(p_{1}, p_{3}, p_{4}, p_{2}, p_{5}\right) \\
& +f^{c_{1} c_{3} c_{x}} f^{c_{5} c_{x} c_{y}} f^{c_{2} c_{4} c_{y}} O_{\nu_{1} \nu_{3} \nu_{5} \nu_{2} \nu_{4}}\left(p_{1}, p_{3}, p_{5}, p_{2}, p_{4}\right)+f^{c_{1} c_{4} c_{x}} f^{c_{2} c_{x} c_{y}} f^{c_{3} c_{5} c_{y}} O_{\nu_{1} \nu_{4} \nu_{2} \nu_{3} \nu_{5}}\left(p_{1}, p_{4}, p_{2}, p_{3}, p_{5}\right) \\
& +f^{c_{1} c_{4} c_{x}} f^{c_{3} c_{x} c_{y}} f^{c_{2} c_{5} c_{y}} O_{\nu_{1} \nu_{4} \nu_{3} \nu_{2} \nu_{5}}\left(p_{1}, p_{4}, p_{3}, p_{2}, p_{5}\right)+f^{c_{1} c_{4} c_{x}} f^{c_{5} c_{x} c_{y}} f^{c_{2} c_{3} c_{y}} O_{\nu_{1} \nu_{4} \nu_{5} \nu_{2} \nu_{3}}\left(p_{1}, p_{4}, p_{5}, p_{2}, p_{3}\right) \\
& +f^{c_{1} c_{5} c_{x}} f^{c_{2} c_{x} c_{y}} f^{c_{3} c_{4} c_{y}} O_{\nu_{1} \nu_{5} \nu_{2} \nu_{3} \nu_{4}}\left(p_{1}, p_{5}, p_{2}, p_{3}, p_{4}\right)+f^{c_{1} c_{5} c_{x}} f^{c_{3} c_{x} c_{y}} f^{c_{2} c_{4} c_{y}} O_{\nu_{1} \nu_{5} \nu_{3} \nu_{2} \nu_{4}}\left(p_{1}, p_{5}, p_{3}, p_{2}, p_{4}\right)
\end{aligned}
$$




$$
\begin{aligned}
& +f^{c_{1} c_{5} c_{x}} f^{c_{4} c_{x} c_{y}} f^{c_{2} c_{3} c_{y}} O_{\nu_{1} \nu_{5} \nu_{4} \nu_{2} \nu_{3}}\left(p_{1}, p_{5}, p_{4}, p_{2}, p_{3}\right)+f^{c_{2} c_{3} c_{x}} f^{c_{1} c_{x} c_{y}} f^{c_{4} c_{5} c_{y}} O_{\nu_{2} \nu_{3} \nu_{1} \nu_{4} \nu_{5}}\left(p_{2}, p_{3}, p_{1}, p_{4}, p_{5}\right) \\
& \left.+f^{c_{2} c_{4} c_{x}} f^{c_{1} c_{x} c_{y}} f^{c_{3} c_{5} c_{y}} O_{\nu_{2} \nu_{4} \nu_{1} \nu_{3} \nu_{5}}\left(p_{2}, p_{4}, p_{1}, p_{3}, p_{5}\right)+f^{c_{2} c_{5} c_{x}} f^{c_{1} c_{x} c_{y}} f^{c_{3} c_{4} c_{y}} O_{\nu_{2} \nu_{5} \nu_{1} \nu_{3} \nu_{4}}\left(p_{2}, p_{5}, p_{1}, p_{3}, p_{4}\right)\right)
\end{aligned}
$$

with

$$
\begin{aligned}
& O_{\nu_{1} \nu_{2} \nu_{3} \nu_{4} \nu_{5}}\left(p_{1}, p_{2}, p_{3}, p_{4}, p_{5}\right)= \\
& \quad \Delta_{\nu_{3}}\left[\Delta_{\nu_{5}} \varepsilon_{\Delta \nu_{1} \nu_{2} \nu_{4}}-\Delta_{\nu_{4}} \varepsilon_{\Delta \nu_{1} \nu_{2} \nu_{5}}\right] \sum_{m=2}^{N-1}\left(\Delta \cdot p_{1}+\Delta \cdot p_{2}\right)^{m-2}\left(-\Delta \cdot p_{4}-\Delta \cdot p_{5}\right)^{N-m-1} \\
& +\tilde{O}_{\nu_{1} \nu_{2} \nu_{3} \nu_{4} \nu_{5}}\left(p_{1}, p_{2}, p_{3}, p_{4}, p_{5}\right)+\tilde{O}_{\nu_{2} \nu_{1} \nu_{3} \nu_{5} \nu_{4}}\left(p_{2}, p_{1}, p_{3}, p_{5}, p_{4}\right)+\tilde{O}_{\nu_{4} \nu_{5} \nu_{3} \nu_{2} \nu_{1}}\left(p_{4}, p_{5}, p_{3}, p_{2}, p_{1}\right) \\
& +\tilde{O}_{\nu_{5} \nu_{4} \nu_{3} \nu_{2} \nu_{1}}\left(p_{5}, p_{4}, p_{3}, p_{2}, p_{1}\right)
\end{aligned}
$$

and

$$
\begin{aligned}
& \tilde{O}_{\nu_{1} \nu_{2} \nu_{3} \nu_{4} \nu_{5}}\left(p_{1}, p_{2}, p_{3}, p_{4}, p_{5}\right)= \\
& -\Delta_{\nu_{2}} \Delta_{\nu_{3}} \Delta_{\nu_{4}}\left[\Delta_{\nu_{5}} \varepsilon_{\Delta p_{5} p_{1} \nu_{1}}+p_{5} . \Delta \varepsilon_{\Delta \nu_{5} \nu_{1} p_{1}}\right] \sum_{m=2}^{N-3} \sum_{n=m+1}^{N-2} \sum_{o=n+1}^{N-1}\left(\Delta \cdot p_{1}\right)^{m-2}\left(\Delta \cdot p_{1}+\Delta \cdot p_{2}\right)^{n-m-1} \\
& \times\left(-\Delta \cdot p_{4}-\Delta \cdot p_{5}\right)^{o-n-1}\left(-\Delta \cdot p_{5}\right)^{N-o-1}+\Delta_{\nu_{3}} \Delta_{\nu_{4}}\left[\Delta_{\nu_{2}} \varepsilon_{\Delta p_{5} \nu_{1} \nu_{5}}-\Delta_{\nu_{1}} \varepsilon_{\left.\Delta p_{5} \nu_{2} \nu_{5}\right]}\right] \\
& \times \sum_{m=2}^{N-2} \sum_{n=m+1}^{N-1}\left(\Delta \cdot p_{1}+\Delta \cdot p_{2}\right)^{m-2}\left(-\Delta \cdot p_{4}-\Delta \cdot p_{5}\right)^{n-m-1}\left(-\Delta \cdot p_{5}\right)^{N-n-1} .
\end{aligned}
$$

All momenta are inflowing and the symbols $f^{a b c}$ denote the structure constants of $\mathrm{SU}\left(N_{c}\right)$. For the sums in the Feynman rule it is understood that the upper summation bound is larger or equal than the lower bound.

Open Access. This article is distributed under the terms of the Creative Commons Attribution License (CC-BY 4.0), which permits any use, distribution and reproduction in any medium, provided the original author(s) and source are credited.

\section{References}

[1] B. Lampe and E. Reya, Spin physics and polarized structure functions, Phys. Rept. 332 (2000) 1 [hep-ph/9810270] [INSPIRE].

[2] S.D. Drell and T.-M. Yan, Partons and their Applications at High-Energies, Annals Phys. 66 (1971) 578 [Annals Phys. 281 (2000) 450] [InSPIRE].

[3] J. Blümlein and H. Böttcher, QCD Analysis of Polarized Deep Inelastic Scattering Data, Nucl. Phys. B 841 (2010) 205 [arXiv:1005.3113] [INSPIRE].

[4] J. Blümlein and H. Böttcher, Higher Twist contributions to the Structure Functions $F_{2}\left(x, Q^{2}\right)$ and $g_{2}\left(x, Q^{2}\right)$, in proceedings of the 20th International Workshop on Deep-Inelastic Scattering and Related Subjects (DIS 2012), Bonn, Germany, 26-30 March 2012, pp. 237-241 [DESY-PROC-2012-02] [arXiv:1207.3170] [INSPIRE].

[5] A. Piccione and G. Ridolfi, Target mass effects in polarized deep inelastic scattering, Nucl. Phys. B 513 (1998) 301 [hep-ph/9707478] [INSPIRE]. 
[6] J. Blümlein and A. Tkabladze, Target mass corrections for polarized structure functions and new sum rules, Nucl. Phys. B 553 (1999) 427 [hep-ph/9812478] [INSPIRE].

[7] D.Y. Bardin, J. Blümlein, P. Christova and L. Kalinovskaya, $O(\alpha)$ QED corrections to neutral current polarized deep-inelastic lepton-nucleon scattering, Nucl. Phys. B 506 (1997) 295 [hep-ph/9612435] [INSPIRE].

[8] A. Arbuzov, D.Y. Bardin, J. Blümlein, L. Kalinovskaya and T. Riemann, Hector 1.00: A Program for the calculation of QED, QCD and electroweak corrections to ep and $l^{ \pm} N$ deep inelastic neutral and charged current scattering, Comput. Phys. Commun. 94 (1996) 128 [hep-ph/9511434] [INSPIRE].

[9] I. Akushevich, A. Ilyichev, N. Shumeiko, A. Soroko and A. Tolkachev, POLARD 2.0 FORTRAN code for the radiative corrections calculation to deep inelastic scattering of polarized particles, Comput. Phys. Commun. 104 (1997) 201 [hep-ph/9706516] [INSPIRE].

[10] J. Blümlein and H. Kawamura, Universal higher order QED corrections to polarized lepton scattering, Nucl. Phys. B 708 (2005) 467 [hep-ph/0409289] [INSPIRE].

[11] H.D. Politzer, Asymptotic Freedom: An Approach to Strong Interactions, Phys. Rept. 14 (1974) 129 [inSPIRE].

[12] A.J. Buras, Asymptotic Freedom in Deep Inelastic Processes in the Leading Order and Beyond, Rev. Mod. Phys. 52 (1980) 199 [INSPIRE].

[13] E. Reya, Perturbative Quantum Chromodynamics, Phys. Rept. 69 (1981) 195 [InSPIRE].

[14] J. Blümlein, The Theory of Deeply Inelastic Scattering, Prog. Part. Nucl. Phys. 69 (2013) 28 [arXiv:1208.6087] [INSPIRE].

[15] D. Boer et al., Gluons and the quark sea at high energies: Distributions, polarization, tomography, arXiv:1108.1713 [INSPIRE].

[16] S. Bethke et al., Workshop on Precision Measurements of alphas, arXiv:1110.0016 [INSPIRE].

[17] S.O. Moch et al., High precision fundamental constants at the TeV scale, arXiv:1405.4781 [INSPIRE].

[18] S. Alekhin, J. Blümlein and S.O. Moch, $\alpha_{s}$ from global fits of parton distribution functions, Mod. Phys. Lett. A 31 (2016) 1630023 [inSPIRE].

[19] S.O. Moch, J.A.M. Vermaseren and A. Vogt, The Three loop splitting functions in QCD: The Nonsinglet case, Nucl. Phys. B 688 (2004) 101 [hep-ph/0403192] [InSPIRE].

[20] J. Blümlein, P. Marquard, C. Schneider and K. Schönwald, The three-loop unpolarized and polarized non-singlet anomalous dimensions from off shell operator matrix elements, Nucl. Phys. B 971 (2021) 115542 [arXiv: 2107.06267] [INSPIRE].

[21] D.J. Gross and F. Wilczek, Asymptotically Free Gauge Theories. I, Phys. Rev. D 8 (1973) 3633 [INSPIRE].

[22] H. Georgi and H.D. Politzer, Electroproduction scaling in an asymptotically free theory of strong interactions, Phys. Rev. D 9 (1974) 416 [InSPIRE].

[23] K. Sasaki, Polarized Electroproduction in Asymptotically Free Gauge Theories, Prog. Theor. Phys. 54 (1975) 1816 [INSPIRE].

[24] M.A. Ahmed and G.G. Ross, Spin-Dependent Deep Inelastic electron Scattering in an Asymptotically Free Gauge Theory, Phys. Lett. B 56 (1975) 385 [INSPIRE]. 
[25] G. Altarelli and G. Parisi, Asymptotic Freedom in Parton Language, Nucl. Phys. B 126 (1977) 298 [inSPIRE].

[26] R. Mertig and W.L. van Neerven, The Calculation of the two loop spin splitting functions $P_{i j}^{(1)}(x)$, Z. Phys. C 70 (1996) 637 [hep-ph/9506451v2] [INSPIRE].

[27] W. Vogelsang, A Rederivation of the spin dependent next-to-leading order splitting functions, Phys. Rev. D 54 (1996) 2023 [hep-ph/9512218] [INSPIRE].

[28] W. Vogelsang, The Spin dependent two loop splitting functions, Nucl. Phys. B 475 (1996) 47 [hep-ph/9603366] [INSPIRE].

[29] Y. Matiounine, J. Smith and W.L. van Neerven, Two loop operator matrix elements calculated up to finite terms for polarized deep inelastic lepton-hadron scattering, Phys. Rev. D 58 (1998) 076002 [hep-ph/9803439] [INSPIRE].

[30] S.O. Moch, J.A.M. Vermaseren and A. Vogt, The Three-Loop Splitting Functions in QCD: The Helicity-Dependent Case, Nucl. Phys. B 889 (2014) 351 [arXiv:1409.5131] [InSPIRE].

[31] A. Behring et al., The Polarized Three-Loop Anomalous Dimensions from On-Shell Massive Operator Matrix Elements, Nucl. Phys. B 948 (2019) 114753 [arXiv:1908.03779] [INSPIRE].

[32] Y. Matiounine, J. Smith and W.L. van Neerven, Two loop operator matrix elements calculated up to finite terms, Phys. Rev. D 57 (1998) 6701 [hep-ph/9801224] [INSPIRE].

[33] J.A. Dixon and J.C. Taylor, Renormalization of Wilson operators in gauge theories, Nucl. Phys. B 78 (1974) 552 [inSPIRE].

[34] H. Kluberg-Stern and J.B. Zuber, Renormalization of NonAbelian Gauge Theories in a Background Field Gauge. 1. Green Functions, Phys. Rev. D 12 (1975) 482 [InSPIRE].

[35] H. Kluberg-Stern and J.B. Zuber, Renormalization of NonAbelian Gauge Theories in a Background Field Gauge. 2. Gauge Invariant Operators, Phys. Rev. D 12 (1975) 3159 [INSPIRE].

[36] S. Sarkar, Mixing of Operators in Wilson Expansions, Nucl. Phys. B 82 (1974) 447 [INSPIRE].

[37] S. Sarkar and H. Strubbe, Anomalous Dimensions in Background Field Gauges, Nucl. Phys. $B 90$ (1975) 45 [INSPIRE].

[38] S.D. Joglekar and B.W. Lee, General Theory of Renormalization of Gauge Invariant Operators, Annals Phys. 97 (1976) 160 [INSPIRE].

[39] S.D. Joglekar, Local Operator Products in Gauge Theories. 1, Annals Phys. 108 (1977) 233 [INSPIRE].

[40] S.D. Joglekar, Local Operator Products in Gauge Theories. 2, Annals Phys. 109 (1977) 210 [INSPIRE].

[41] R. Hamberg and W.L. van Neerven, The Correct renormalization of the gluon operator in a covariant gauge, Nucl. Phys. B 379 (1992) 143 [inSPIRE].

[42] R. Hamberg, Second order gluonic contributions to physical quantities, Ph.D. Thesis, Leiden University, Leiden The Netherlands (1991).

[43] J.C. Collins and R.J. Scalise, The Renormalization of composite operators in Yang-Mills theories using general covariant gauge, Phys. Rev. D 50 (1994) 4117 [hep-ph/9403231] [INSPIRE]. 
[44] B.W. Harris and J. Smith, Anomalous dimension of the gluon operator in pure Yang-Mills theory, Phys. Rev. D 51 (1995) 4550 [hep-ph/9409405] [INSPIRE].

[45] J. Blümlein, G. Falcioni and A. De Freitas, The Complete $O\left(\alpha_{s}^{2}\right)$ Non-Singlet Heavy Flavor Corrections to the Structure Functions $g_{1,2}^{e p}\left(x, Q^{2}\right), F_{1,2, L}^{e p}\left(x, Q^{2}\right), F_{1,2,3}^{\nu(\bar{\nu}}\left(x, Q^{2}\right)$ and the Associated Sum Rules, Nucl. Phys. B 910 (2016) 568 [arXiv:1605.05541] [inSPIRE].

[46] A. Behring, J. Blümlein, A. De Freitas, A. von Manteuffel and C. Schneider, The 3-Loop Non-Singlet Heavy Flavor Contributions to the Structure Function $g_{1}\left(x, Q^{2}\right)$ at Large Momentum Transfer, Nucl. Phys. B 897 (2015) 612 [arXiv:1504.08217] [InSPIRE].

[47] J. Blümlein and M. Saragnese, The $N^{3} L O$ scheme-invariant $Q C D$ evolution of the non-singlet structure functions $F_{2}^{N S}\left(x, Q^{2}\right)$ and $g_{1}^{N S}\left(x, Q^{2}\right)$, Phys. Lett. B 820 (2021) 136589 [arXiv:2107.01293] [INSPIRE].

[48] S.A. Larin, The Renormalization of the axial anomaly in dimensional regularization, Phys. Lett. B 303 (1993) 113 [hep-ph/9302240] [INSPIRE].

[49] J. Blümlein and C. Schneider, The Method of Arbitrarily Large Moments to Calculate Single Scale Processes in Quantum Field Theory, Phys. Lett. B 771 (2017) 31 [arXiv: 1701.04614] [INSPIRE].

[50] S.O. Moch, J.A.M. Vermaseren and A. Vogt, $O n \gamma_{5}$ in higher-order QCD calculations and the NNLO evolution of the polarized valence distribution, Phys. Lett. B 748 (2015) 432 [arXiv: 1506. 04517] [INSPIRE].

[51] F. Yndurain, The Theory of Quark and Gluon, Springer, Berlin Germany (2006).

[52] I. Bierenbaum, J. Blümlein and S. Klein, Mellin Moments of the $O\left(\alpha_{s}^{3}\right)$ Heavy Flavor Contributions to unpolarized Deep-Inelastic Scattering at $Q^{2} \gg m^{2}$ and Anomalous Dimensions, Nucl. Phys. B 820 (2009) 417 [arXiv:0904.3563] [INSPIRE].

[53] G. 't Hooft and M.J.G. Veltman, Regularization and Renormalization of Gauge Fields, Nucl. Phys. B 44 (1972) 189 [INSPIRE].

[54] D.A. Akyeampong and R. Delbourgo, Dimensional regularization, abnormal amplitudes and anomalies, Nuovo Cim. A 17 (1973) 578 [INSPIRE].

[55] D.A. Akyeampong and R. Delbourgo, Dimensional regularization and PCAC, Nuovo Cim. A 18 (1973) 94 [INSPIRE].

[56] D.A. Akyeampong and R. Delbourgo, Anomalies via dimensional regularization, Nuovo Cim. A 19 (1974) 219 [inSPIRE].

[57] P. Breitenlohner and D. Maison, Dimensionally Renormalized Green's Functions for Theories with Massless Particles. 1, Commun. Math. Phys. 52 (1977) 39 [InSPIRE].

[58] P. Breitenlohner and D. Maison, Dimensionally Renormalized Green's Functions for Theories with Massless Particles. 2, Commun. Math. Phys. 52 (1977) 55 [inSPIRE].

[59] D. Kreimer, The $\gamma_{5}$-problem and anomalies: A Clifford algebra approach, Phys. Lett. B 237 (1990) 59 [INSPIRE].

[60] J.G. Körner, D. Kreimer and K. Schilcher, A Practicable $\gamma_{5}$-scheme in dimensional regularization, Z. Phys. C 54 (1992) 503 [InSPIRE].

[61] D. Kreimer, Dimensionale Regularisierung im Standardmodell, Ph.D. Thesis, Johannes Gutenberg University Mainz, Mainz Germany (1992).

[62] D. Kreimer, The Role of $\gamma_{5}$ in dimensional regularization, hep-ph/9401354 [INSPIRE]. 
[63] T.L. Trueman, Spurious anomalies in dimensional renormalization, Z. Phys. C 69 (1996) 525 [hep-ph/9504315] [INSPIRE].

[64] E. Kraus, Renormalization of the Electroweak Standard Model to All Orders, Annals Phys. 262 (1998) 155 [hep-th/9709154] [INSPIRE].

[65] S. Weinzierl, Equivariant dimensional regularization, hep-ph/9903380 [INSPIRE].

[66] D. Stöckinger, Methoden zur Renormierung supersymmetrischer Eichtheorien in der Wess-Zumino-Eichung und deren Anwendung, Ph.D. Thesis, Karlsruhe Institute of Technology, Karlsruhe Germany (2001) [INSPIRE].

[67] F. Jegerlehner, Facts of life with $\gamma_{5}$, Eur. Phys. J. C 18 (2001) 673 [hep-th/0005255] [INSPIRE].

[68] H. Bélusca-Maïto, A. Ilakovac, P. Kühler, M. Mađor-Božinović and D. Stöckinger, Two-loop application of the Breitenlohner-Maison/'t Hooft-Veltman scheme with non-anticommuting $\gamma_{5}$ : full renormalization and symmetry-restoring counterterms in an Abelian chiral gauge theory, JHEP 11 (2021) 159 [arXiv:2109.11042] [INSPIRE].

[69] J. Blümlein, V. Ravindran and W.L. van Neerven, On the Drell-Levy-Yan relation to $O\left(\alpha_{s}^{2}\right)$, Nucl. Phys. B 586 (2000) 349 [hep-ph/0004172] [inSPIRE].

[70] J. Blümlein and H. Böttcher, QCD analysis of polarized deep inelastic data and parton distributions, Nucl. Phys. B 636 (2002) 225 [hep-ph/0203155] [INSPIRE].

[71] G. Altarelli, R.K. Ellis and G. Martinelli, Leptoproduction and Drell-Yan Processes Beyond the Leading Approximation in Chromodynamics, Nucl. Phys. B 143 (1978) 521 [Erratum ibid. 146 (1978) 544] [INSPIRE].

[72] E.B. Zijlstra and W.L. van Neerven, Order- $\alpha_{s}^{2}$ corrections to the polarized structure function $g_{1}\left(x, Q^{2}\right)$, Nucl. Phys. B 417 (1994) 61 [Erratum ibid. 426 (1994) 245] [Erratum ibid. 773 (2007) 105] [Erratum ibid. 501 (1997) 599] [INSPIRE].

[73] D.J. Gross and F. Wilczek, Ultraviolet Behavior of NonAbelian Gauge Theories, Phys. Rev. Lett. 30 (1973) 1343 [INSPIRE].

[74] H.D. Politzer, Reliable Perturbative Results for Strong Interactions?, Phys. Rev. Lett. 30 (1973) 1346 [INSPIRE].

[75] W.E. Caswell, Asymptotic Behavior of NonAbelian Gauge Theories to Two Loop Order, Phys. Rev. Lett. 33 (1974) 244 [INSPIRE].

[76] D.R.T. Jones, Two Loop Diagrams in Yang-Mills Theory, Nucl. Phys. B 75 (1974) 531 [INSPIRE].

[77] O.V. Tarasov, A.A. Vladimirov and A.Y. Zharkov, The Gell-Mann-Low Function of QCD in the Three Loop Approximation, Phys. Lett. B 93 (1980) 429 [INSPIRE].

[78] S.A. Larin and J.A.M. Vermaseren, The Three loop QCD $\beta$-function and anomalous dimensions, Phys. Lett. B 303 (1993) 334 [hep-ph/9302208] [INSPIRE].

[79] T. van Ritbergen, J.A.M. Vermaseren and S.A. Larin, The Four loop $\beta$-function in quantum chromodynamics, Phys. Lett. B 400 (1997) 379 [hep-ph/9701390] [INSPIRE].

[80] M. Czakon, The Four-loop QCD $\beta$-function and anomalous dimensions, Nucl. Phys. B 710 (2005) 485 [hep-ph/0411261] [INSPIRE].

[81] P.A. Baikov, K.G. Chetyrkin and J.H. Kühn, Five-Loop Running of the QCD coupling constant, Phys. Rev. Lett. 118 (2017) 082002 [arXiv:1606. 08659] [INSPIRE]. 
[82] F. Herzog, B. Ruijl, T. Ueda, J.A.M. Vermaseren and A. Vogt, The five-loop $\beta$-function of Yang-Mills theory with fermions, JHEP 02 (2017) 090 [arXiv: 1701.01404] [INSPIRE].

[83] T. Luthe, A. Maier, P. Marquard and Y. Schröder, The five-loop $\beta$-function for a general gauge group and anomalous dimensions beyond Feynman gauge, JHEP 10 (2017) 166 [arXiv:1709.07718] [INSPIRE].

[84] K.G. Chetyrkin, G. Falcioni, F. Herzog and J.A.M. Vermaseren, Five-loop renormalisation of QCD in covariant gauges, JHEP 10 (2017) 179 [Addendum JHEP 12 (2017) 006] [arXiv:1709.08541] [INSPIRE].

[85] K.G. Chetyrkin, Four-loop renormalization of QCD: Full set of renormalization constants and anomalous dimensions, Nucl. Phys. B 710 (2005) 499 [hep-ph/0405193] [INSPIRE].

[86] E. Egorian and O.V. Tarasov, Two Loop Renormalization of the QCD in an Arbitrary Gauge, Teor. Mat. Fiz. 41 (1979) 26 [Theor. Math. Phys. 41 (1979) 863] [InSPIRE].

[87] T. Luthe, A. Maier, P. Marquard and Y. Schröder, Five-loop quark mass and field anomalous dimensions for a general gauge group, JHEP 01 (2017) 081 [arXiv:1612.05512] [INSPIRE].

[88] J. Blümlein, P. Marquard, C. Schneider and K. Schönwald, The Two-Loop QCD Operator Matrix Elements to Finite Terms, DESY-21-028 (2021).

[89] J. Blümlein and N. Kochelev, On the twist-2 and twist-3 contributions to the spin dependent electroweak structure functions, Nucl. Phys. B 498 (1997) 285 [hep-ph/9612318] [InSPIRE].

[90] P. Nogueira, Automatic Feynman graph generation, J. Comput. Phys. 105 (1993) 279 [INSPIRE].

[91] J.A.M. Vermaseren, New features of FORM, math-ph/0010025 [INSPIRE].

[92] M. Tentyukov and J.A.M. Vermaseren, The Multithreaded version of FORM, Comput. Phys. Commun. 181 (2010) 1419 [hep-ph/0702279] [INSPIRE].

[93] T. van Ritbergen, A.N. Schellekens and J.A.M. Vermaseren, Group theory factors for Feynman diagrams, Int. J. Mod. Phys. A 14 (1999) 41 [hep-ph/9802376] [InSPIRE].

[94] J. Ablinger, J. Blümlein, S. Klein and C. Schneider, Modern Summation Methods and the Computation of 2- and 3-loop Feynman Diagrams, Nucl. Phys. B Proc. Suppl. 205-206 (2010) 110 [arXiv: 1006.4797] [INSPIRE].

[95] J. Blümlein, A. Hasselhuhn and C. Schneider, Evaluation of Multi-Sums for Large Scale Problems, PoS RAD COR 2011 (2011) 032 [arXiv:1202.4303] [INSPIRE].

[96] C. Schneider, Symbolic Summation in Difference Fields and Its Application in Particle Physics, Computeralgebra-Rundbrief 53 (2013) 8.

[97] C. Schneider, Modern Summation Methods for Loop Integrals in Quantum Field Theory: The Packages Sigma, EvaluateMultiSums and SumProduction, J. Phys. Conf. Ser. 523 (2014) 012037 [arXiv:1310.0160] [INSPIRE].

[98] P. Marquard and D. Seidel, The Crusher algorithm, unpublished.

[99] J. Blümlein, P. Marquard and C. Schneider, A refined machinery to calculate large moments from coupled systems of linear differential equations, PoS RADCOR2019 (2019) 078 [arXiv: 1912.04390] [INSPIRE].

[100] M. Kauers, Guessing Handbook, RISC, Johannes Kepler University Linz, Linz Austria (2009) [Technical Report RISC 09-07]. 
[101] J. Blümlein, M. Kauers, S. Klein and C. Schneider, Determining the closed forms of the $O\left(\alpha_{s}^{3}\right)$ anomalous dimensions and Wilson coefficients from Mellin moments by means of computer algebra, Comput. Phys. Commun. 180 (2009) 2143 [arXiv:0902.4091] [INSPIRE].

[102] Sage, (2022) http://www.sagemath.org/.

[103] M. Kauers, M. Jaroschek and F. Johansson, Ore Polynomials in Sage, in Computer Algebra and Polynomials, Lecture Notes in Computer Science 8942, J. Gutierrez, J. Schicho and M. Weimann eds., Springer, Cham Switzerland (2015), pp. 105-125 [arXiv: 1306 .4263].

[104] C. Schneider, Symbolic Summation Assists Combinatorics, Sémin. Lothar. Comb. 56 (2007) B56b.

[105] C. Schneider, Simplifying Multiple Sums in Difference Fields, in Computer Algebra in Quantum Field Theory: Integration, Summation and Special Functions, Texts and Monographs in Symbolic Computation, C. Schneider and J. Blümlein eds., Springer, Wien Austria (2013), pp. 325-360 [arXiv:1304.4134].

[106] J.A.M. Vermaseren, Harmonic sums, Mellin transforms and integrals, Int. J. Mod. Phys. A 14 (1999) 2037 [hep-ph/9806280] [INSPIRE].

[107] J. Blümlein and S. Kurth, Harmonic sums and Mellin transforms up to two loop order, Phys. Rev. D 60 (1999) 014018 [hep-ph/9810241] [INSPIRE].

[108] E. Remiddi and J.A.M. Vermaseren, Harmonic polylogarithms, Int. J. Mod. Phys. A 15 (2000) 725 [hep-ph/9905237] [INSPIRE].

[109] J. Ablinger, J. Blümlein and C. Schneider, Generalized Harmonic, Cyclotomic, and Binomial Sums, their Polylogarithms and Special Numbers, J. Phys. Conf. Ser. 523 (2014) 012060 [arXiv: 1310.5645] [INSPIRE].

[110] J. Ablinger, The package HarmonicSums: Computer Algebra and Analytic aspects of Nested Sums, PoS LL2014 (2014) 019 [arXiv:1407.6180] [InSPIRE].

[111] J. Ablinger, A Computer Algebra Toolbox for Harmonic Sums Related to Particle Physics, Ph.D. Thesis, Johannes Kepler University Linz, Linz Austria (2009) [arXiv:1011.1176] [INSPIRE].

[112] J. Ablinger, Computer Algebra Algorithms for Special Functions in Particle Physics, Ph.D. Thesis, Linz University, Linz Austria (2012) [arXiv: 1305. 0687] [INSPIRE].

[113] J. Ablinger, Inverse Mellin Transform of Holonomic Sequences, PoS LL2016 (2016) 067 [INSPIRE].

[114] J. Ablinger, Discovering and Proving Infinite Binomial Sums Identities, Exper. Math. 26 (2016) 62 [arXiv:1507.01703] [INSPIRE].

[115] J. Ablinger, Computing the Inverse Mellin Transform of Holonomic Sequences using Kovacic's Algorithm, PoS RADCOR2017 (2018) 001 [arXiv:1801.01039] [INSPIRE].

[116] J. Ablinger, Discovering and Proving Infinite Pochhammer Sum Identities, arXiv:1902.11001 [INSPIRE].

[117] J. Ablinger, An Improved Method to Compute the Inverse Mellin Transform of Holonomic Sequences, PoS LL2018 (2018) 063 [INSPIRE].

[118] J. Ablinger, J. Blümlein and C. Schneider, Harmonic Sums and Polylogarithms Generated by Cyclotomic Polynomials, J. Math. Phys. 52 (2011) 102301 [arXiv:1105.6063] [INSPIRE]. 
[119] J. Ablinger, J. Blümlein and C. Schneider, Analytic and Algorithmic Aspects of Generalized Harmonic Sums and Polylogarithms, J. Math. Phys. 54 (2013) 082301 [arXiv:1302.0378] [INSPIRE].

[120] J. Ablinger, J. Blümlein, C.G. Raab and C. Schneider, Iterated Binomial Sums and their Associated Iterated Integrals, J. Math. Phys. 55 (2014) 112301 [arXiv:1407.1822] [INSPIRE].

[121] J. Blümlein, Algebraic relations between harmonic sums and associated quantities, Comput. Phys. Commun. 159 (2004) 19 [hep-ph/0311046] [inSPIRE].

[122] J. Blümlein, D.J. Broadhurst and J.A.M. Vermaseren, The Multiple Zeta Value Data Mine, Comput. Phys. Commun. 181 (2010) 582 [arXiv:0907.2557] [INSPIRE].

[123] J. Blümlein, Structural Relations of Harmonic Sums and Mellin Transforms up to Weight $w=5$, Comput. Phys. Commun. 180 (2009) 2218 [arXiv:0901.3106] [InSPIRE].

[124] J.L. Lagrange, Nouvelles recherches sur la nature et la propagation du son, in Euvres de Lagrange. Tome Premier, Les Soins de M.J.-A. Serret et G. Darboux, Paris France (1867), pp. 151-316 [Miscellanea Taurinensis II (1762) 11].

[125] C.F. Gauß, Theoria attractionis corporum sphaeroidicorum ellipticorum homogeneorum. Methodo novo tractate, in Commentationes Societas Scientiarum Gottingensis Recentiores. Volumen III, H. Dieterich (1813), pp. 5-7, republished in Werke. Fünfter Band, Springer, pp. 3-22 [https://doi.org/10.1007/978-3-642-49319-5_1].

[126] G. Green, Essay on the Mathematical Theory of Electricity and Magnetism, Nottingham U.K. (1828), pp. $1-115$.

[127] M. Ostrogradski, Note sur une intégrale qui se rencontre dans le calcul de l'attraction des sphéroïdes, Mem. Acad. Sci. St. Petersb. 1 (1831) 39.

[128] S. Laporta, High precision calculation of multiloop Feynman integrals by difference equations, Int. J. Mod. Phys. A 15 (2000) 5087 [hep-ph/0102033] [INSPIRE].

[129] M. Karr, Summation in finite terms, J. ACM 28 (1981) 305.

[130] M. Bronstein, On Solutions of Linear Ordinary Difference Equations in their Coefficient Field, J. Symbolic Comput. 29 (2000) 841.

[131] C. Schneider, Symbolic Summation in Difference Fields, Ph.D. Thesis, RISC, Johannes Kepler University Linz, Linz Austria (2001) [Technical Report 01-17].

[132] C. Schneider, Difference Equations in $\Pi \Sigma$-Extensions, An. Univ. Vest Timis. Ser. Mat.-Inform. 42 (2004) 163.

[133] C. Schneider, Solving parameterized linear difference equations in terms of indefinite nested sums and products, J. Differ. Equ. Appl. 11 (2005) 799.

[134] C. Schneider, Degree Bounds to Find Polynomial Solutions of Parameterized Linear

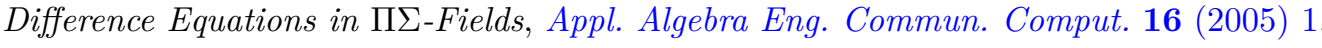

[135] C. Schneider, Simplifying sums in $\Pi \Sigma^{*}$-extensions, J. Algebra Appl. 6 (2007) 415.

[136] C. Schneider, A Symbolic Summation Approach to Find Optimal Nested Sum Representations, Clay Math. Proc. 12 (2010) 285 [arXiv:0904.2323] [INSPIRE].

[137] C. Schneider, Parameterized Telescoping Proves Algebraic Independence of Sums, arXiv:0808.2596 [INSPIRE]. 
[138] C. Schneider, Fast Algorithms for Refined Parameterized Telescoping in Difference Fields, in Computer Algebra and Polynomials, Applications of Algebra and Number Theory, Lecture Notes in Computer Science 8942, J. Gutierrez, J. Schicho and M. Weimann eds., Springer, Cham Switzerland (2015), pp. 157-191 [arXiv:1307.7887].

[139] C. Schneider, A difference ring theory for symbolic summation, J. Symbolic Comput. 72 (2016) 82 [arXiv:1408.2776].

[140] C. Schneider, Summation Theory II: Characterizations of $R \Pi \Sigma^{*}$-extensions and algorithmic aspects, J. Symbolic Comput. 80 (2017) 616 [arXiv:1603.04285].

[141] S.A. Abramov, M. Bronstein and M. Petkovšek, On rational and hypergeometric solutions of linear ordinary difference equations in $\Pi \Sigma^{*}$-field extensions, J. Symbolic Comput. 107 (2021) 23 [arXiv: 2005.04944].

[142] K.G. Chetyrkin and F.V. Tkachov, Integration by Parts: The Algorithm to Calculate $\beta$-functions in 4 Loops, Nucl. Phys. B 192 (1981) 159 [INSPIRE].

[143] F.V. Tkachov, A Theorem on Analytical Calculability of Four Loop Renormalization Group Functions, Phys. Lett. B 100 (1981) 65 [INSPIRE].

[144] F.V. Tkachov, An algorithm for calculating multiloop integrals, Theor. Math. Phys. 56 (1983) 866 [Teor. Mat. Fiz. 56 (1983) 350] [INSPIRE].

[145] T. Gehrmann, E.W.N. Glover, T. Huber, N. Ikizlerli and C. Studerus, Calculation of the quark and gluon form factors to three loops in QCD, JHEP 06 (2010) 094 [arXiv: 1004.3653] [INSPIRE].

[146] R.N. Lee, A.V. Smirnov and V.A. Smirnov, Dimensional recurrence relations: an easy way to evaluate higher orders of expansion in $\epsilon$, Nucl. Phys. B Proc. Suppl. 205-206 (2010) 308 [arXiv: 1005.0362] [INSPIRE].

[147] R.N. Lee and V.A. Smirnov, Analytic $\epsilon$-expansions of Master Integrals Corresponding to Massless Three-Loop Form Factors and Three-Loop g - 2 up to Four-Loop Transcendentality Weight, JHEP 02 (2011) 102 [arXiv:1010.1334] [INSPIRE].

[148] T. Gehrmann and E. Remiddi, Numerical evaluation of two-dimensional harmonic polylogarithms, Comput. Phys. Commun. 144 (2002) 200 [hep-ph/0111255] [InSPIRE].

[149] J. Vollinga and S. Weinzierl, Numerical evaluation of multiple polylogarithms, Comput. Phys. Commun. 167 (2005) 177 [hep-ph/0410259] [INSPIRE].

[150] J. Ablinger, J. Blümlein, M. Round and C. Schneider, Numerical Implementation of Harmonic Polylogarithms to Weight $w=8$, Comput. Phys. Commun. 240 (2019) 189 [arXiv: 1809.07084] [INSPIRE].

[151] J. Ablinger, J. Blümlein, P. Marquard, N. Rana and C. Schneider, Automated Solution of First Order Factorizable Systems of Differential Equations in One Variable, Nucl. Phys. B 939 (2019) 253 [arXiv: 1810.12261] [INSPIRE].

[152] J. Ablinger and J. Blümlein, Harmonic Sums, Polylogarithms, Special Numbers, and Their Generalizations, in Computer Algebra in Quantum Field Theory. Integration, Summation and Special Functions, Texts \& Monographs in Symbolic Computation, C. Schneider and J. Blümlein eds., Springer, Wien Austria (2013), pp. 1-32 [arXiv:1304.7071] [INSPIRE].

[153] J. Blümlein, Analytic continuation of Mellin transforms up to two loop order, Comput. Phys. Commun. 133 (2000) 76 [hep-ph/0003100] [INSPIRE]. 
[154] J. Blümlein and S.O. Moch, Analytic continuation of the harmonic sums for the 3-loop anomalous dimensions, Phys. Lett. B 614 (2005) 53 [hep-ph/0503188] [INSPIRE].

[155] A.V. Kotikov and V.N. Velizhanin, Analytic continuation of the Mellin moments of deep inelastic structure functions, hep-ph/0501274 [INSPIRE].

[156] J. Blümlein, Structural Relations of Harmonic Sums and Mellin Transforms at Weight $w=6$, Clay Math. Proc. 12 (2010) 167 [arXiv:0901.0837] [inSPIRE].

[157] W.L. van Neerven, Fortran - code for the Wilson coefficients of the structure functions $g_{1}\left(x, Q^{2}\right)$ to two-loop order, unpublished.

[158] I. Bierenbaum et al., $O\left(\alpha_{s}^{2}\right)$ Polarized Heavy Flavor Production in Deep-Inelastic Scattering at $Q^{2} \gg m^{2}$, DESY-15-004 (2015).

[159] A.D. Watson, Spin Spin Asymmetries in Inclusive Muon Proton Charm Production, Z. Phys. C 12 (1982) 123 [InSPIRE].

[160] M. Glück, E. Reya and W. Vogelsang, Determination of spin dependent parton distributions in polarized leptoproduction of jets and heavy quarks, Nucl. Phys. B 351 (1991) 579 [INSPIRE].

[161] W. Vogelsang, The Gluonic contribution to $g_{1}^{p}\left(x, Q^{2}\right)$ in the parton model, Z. Phys. C 50 (1991) 275 [INSPIRE].

[162] J.A. Gracey, Anomalous dimensions of operators in polarized deep inelastic scattering at $O\left(1 / N_{f}\right)$, Nucl. Phys. B 480 (1996) 73 [hep-ph/9609301] [InSPIRE].

[163] J.F. Bennett and J.A. Gracey, Anomalous dimension of gluonic operator in polarized deep inelastic scattering at $O\left(1 / N_{f}\right)$, Phys. Lett. B 432 (1998) 209 [hep-ph/9803446] [INSPIRE].

[164] J. Blümlein, QCD evolution of structure functions at small $x$, in Lecture Notes in Physics 546, Springer (2000), pp. 42-57 [hep-ph/9909449] [INSPIRE].

[165] J. Bartels, B.I. Ermolaev and M.G. Ryskin, Flavor singlet contribution to the structure function $g_{1}$ at small x, Z. Phys. C 72 (1996) 627 [hep-ph/9603204] [INSPIRE].

[166] J. Blümlein and A. Vogt, The Singlet contribution to the structure function $g_{1}\left(x, Q^{2}\right)$ at small x, Phys. Lett. B 386 (1996) 350 [hep-ph/9606254] [INSPIRE].

[167] A. Vogt, S.O. Moch, M. Rogal and J.A.M. Vermaseren, Towards the NNLO evolution of polarised parton distributions, Nucl. Phys. B Proc. Suppl. 183 (2008) 155 [arXiv: 0807.1238] [INSPIRE].

[168] Y. Kiyo, J. Kodaira and H. Tochimura, Does leading ln $x$ resummation predict the rise of $g_{1}$ at small x?, Z. Phys. C 74 (1997) 631 [hep-ph/9701365] [INSPIRE].

[169] J. Bartels, B.I. Ermolaev and M.G. Ryskin, Nonsinglet contributions to the structure function $g_{1}$ at small $x$, Z. Phys. C $\mathbf{7 0}$ (1996) 273 [hep-ph/9507271] [INSPIRE].

[170] R. Kirschner and L.N. Lipatov, Double Logarithmic Asymptotics and Regge Singularities of Quark Amplitudes with Flavor Exchange, Nucl. Phys. B 213 (1983) 122 [InSPIRE].

[171] J. Blümlein and A. Vogt, On the behavior of nonsinglet structure functions at small $x$, Phys. Lett. B 370 (1996) 149 [hep-ph/9510410] [INSPIRE].

[172] S. Wandzura and F. Wilczek, Sum Rules for Spin Dependent Electroproduction: Test of Relativistic Constituent Quarks, Phys. Lett. B 72 (1977) 195 [INSPIRE]. 
[173] J. Ablinger, J. Blümlein, A. De Freitas, M. Saragnese, C. Schneider and K. Schönwald, The three-loop polarized pure singlet operator matrix element with two different masses, Nucl. Phys. B 952 (2020) 114916 [arXiv:1911.11630] [INSPIRE].

[174] J. Ablinger et al., The three-loop single mass polarized pure singlet operator matrix element, Nucl. Phys. B 953 (2020) 114945 [arXiv:1912.02536] [InSPIRE].

[175] A. Behring, J. Blümlein, A. De Freitas, A. von Manteuffel, K. Schönwald and C. Schneider, The polarized transition matrix element $A_{g q}(N)$ of the variable flavor number scheme at $O\left(\alpha_{s}^{3}\right)$, Nucl. Phys. B 964 (2021) 115331 [arXiv:2101.05733] [INSPIRE].

[176] J. Blümlein, A. De Freitas, M. Saragnese, C. Schneider and K. Schönwald, Logarithmic contributions to the polarized $O\left(\alpha_{s}^{3}\right)$ asymptotic massive Wilson coefficients and operator matrix elements in deeply inelastic scattering, Phys. Rev. D 104 (2021) 034030 [arXiv: 2105. 09572] [INSPIRE].

[177] J. Ablinger et al., The two-mass contribution to the three-loop polarized gluonic operator matrix element $A_{g g, Q}^{(3)}$, Nucl. Phys. B 955 (2020) 115059 [arXiv: 2004.08916] [INSPIRE].

[178] J. Ablinger et al., The unpolarized single mass three-loop operator matrix element $A_{g g, Q}$, DESY-15-112 (2015).

[179] J. Ablinger et al., The polarized three-loop operator matrix element $A_{g g, Q}$, DESY-20-053 (2020).

[180] J. Blümlein et al., Heavy Flavor Wilson Coefficients in Deep-Inelastic Scattering: Recent Results, PoS QCDEV2017 (2017) 031 [arXiv:1711.07957] [INSPIRE]. 